PREPARED FOR SUBMISSION TO JHEP

\title{
Supergravity as Generalised Geometry I: Type II Theories
}

\author{
André Coimbra ${ }^{1}$, Charles Strickland-Constable ${ }^{2}$ and Daniel Waldram ${ }^{3}$ \\ Department of Physics, Imperial College London \\ Prince Consort Road, London, SW' 2AZ, UK
}

\begin{abstract}
We reformulate ten-dimensional type II supergravity as a generalised geometrical analogue of Einstein gravity, defined by an $O(9,1) \times O(1,9) \subset O(10,10) \times \mathbb{R}^{+}$ structure on the generalised tangent space. Using the notion of generalised connection and torsion, we introduce the analogue of the Levi-Civita connection, and derive the corresponding tensorial measures of generalised curvature. We show how, to leading order in the fermion fields, these structures allow one to rewrite the action, equations of motion and supersymmetry variations in a simple, manifestly $\operatorname{Spin}(9,1) \times \operatorname{Spin}(1,9)$ covariant form. The same formalism also describes $d$-dimensional compactifications to flat space.
\end{abstract}

\footnotetext{
${ }^{1}$ a.coimbra08@imperial.ac.uk

${ }^{2}$ charles.strickland-constable08@imperial.ac.uk

${ }^{3}$ d.waldram@imperial.ac.uk
} 


\section{Contents}

1 Introduction $\quad 2$

2 Type II supergravity $\quad 4$

2.1 Degrees of freedom, equations of motion and supersymmetry 5

$\begin{array}{ll}2.2 \text { Bosonic symmetries } & 7\end{array}$

$3 \quad O(d, d) \times \mathbb{R}^{+}$generalised geometry $\quad \mathbf{9}$

3.1 Generalised structure bundle 9

3.2 Generalised tensors and spinors and split frames 10

3.3 The Dorfman derivative, Courant bracket and exterior derivative $\quad 12$

3.4 Generalised $O(d, d) \times \mathbb{R}^{+}$connections and torsion 14

$\begin{array}{lll}3.4 .1 & \text { Generalised connections } & 15\end{array}$

$\begin{array}{lll}3.4 .2 & \text { Generalised torsion } & 16\end{array}$

3.4.3 The absence of generalised curvature 17

$4 O(p, q) \times O(q, p)$ structures and torsion-free connections $\quad 18$

$4.1 O(p, q) \times O(q, p)$ structures and the generalised metric $\quad 18$

4.2 Torsion-free, compatible connections 20

4.3 Unique operators and generalised $O(p, q) \times O(q, p)$ curvatures 23

5 Type II supergravity as $O(9,1) \times O(1,9)$ generalised gravity 25

5.1 NSNS and fermionic supergravity fields 25

5.2 RR fields 26

5.3 Supersymmetry variations 28

5.4 Equations of motion 29

6 Conclusions and discussion $\quad 31$

$\begin{array}{ll}\text { A Supergravity Conventions } & 32\end{array}$

B Metric structures, torsion and the Levi-Civita connection 33 


\section{Introduction}

Generalised geometry $[1,2]$ is the study of structures on a generalised tangent space $E \simeq T M \oplus T^{*} M$. Local diffeomorphism invariance is replaced by a larger group that also includes the gauge transformations of the NSNS two-form $B$ and there is a natural $O(d, d)$ structure on $E$, forming a Courant algebroid [3]. Since it was first applied to supersymmetric type II backgrounds [4-6] and string sigma models [7], it has been clear that it is closely connected to the geometry of supergravity.

In this paper we show that ten-dimensional type IIA and IIB supergravity theories, to leading order in the fermions, can be formulated precisely as generalised geometrical analogues of Einstein gravity. The theory has manifest local $\operatorname{Spin}(9,1) \times \operatorname{Spin}(1,9)$ symmetry and admits a natural analogue of the Levi-Civita connection. Remarkably both bosonic and fermionic equations of motion and all the supersymmetry variations take a simple form in terms of this generalised connection. Although we will focus on the ten-dimensional case, the same formalism, with a local $\operatorname{Spin}(d) \times \operatorname{Spin}(d)$ symmetry, also describes the type II fields restricted to a $d$-dimensional manifold $M$ used to compactify the ten-dimensional theory to flat space.

Interestingly, such rewritings in terms of generalised geometry appear not to be restricted to type II theories. In a forthcoming companion paper [8], we define the corresponding structures in the $E_{d(d)}$ version of generalised geometry $[9,10]$ relevant to restrictions of eleven-dimensional supergravity to a $d$-dimensional manifold.

Let us start by briefly summarizing our construction and results. We slightly extend the action on the generalised tangent space to a conformal $O(10,10) \times \mathbb{R}^{+}$structure. The NSNS fields then define an $O(9,1) \times O(1,9)$ substructure. The RR field strengths $F$ are described by a $\operatorname{Spin}(10,10)$ spinor, while the fermions transform in particular spinor representations of the two $\operatorname{Spin}(9,1)$ groups. The supergravity is described as an analogue of conventional gravity, where $O(10,10) \times \mathbb{R}^{+}$and $O(9,1) \times O(1,9)$ play the role of the $G L(d, \mathbb{R})$ and $O(d)$ actions of the frame bundle respectively, and the diffeomorphism group is replaced by $G_{\mathrm{NS}}$, an extension by NSNS $B$-field transformations.

The central object in the construction is the analogue of the Levi-Civita connection, a generalised connection $D$ that is both compatible with the $O(9,1) \times O(1,9)$ structure and torsion-free in a generalised sense. Interestingly this connection is not unique. However, using the $O(9,1) \times O(1,9)$ structure one can contract indices to construct unique expressions. Using $D$, the dynamics and symmetries of the supergravity theories then can be written in a simple $\operatorname{Spin}(9,1) \times \operatorname{Spin}(1,9)$ covariant form. For example, the supersymmetry variations of the gravitini and dilatini can be written as

$$
\begin{array}{ll}
\delta \psi_{\bar{a}}^{+}=D_{\bar{a}} \epsilon^{+}+\frac{1}{16} F_{\#} \gamma_{\bar{a}} \epsilon^{-}, & \delta \rho^{+}=\gamma^{a} D_{a} \epsilon^{+}, \\
\delta \psi_{a}^{-}=D_{a} \epsilon^{-}+\frac{1}{16} F_{\#}^{T} \gamma_{a} \epsilon^{+}, & \delta \rho^{-}=\gamma^{\bar{a}} D_{\bar{a}} \epsilon^{-} .
\end{array}
$$


Here \pm and $a$ and $\bar{a}$ refer to spinors and vector indices respectively of the two $\operatorname{Spin}(9,1)$ groups while $F_{\#}$ denotes the $\mathrm{RR}$ fields viewed as a $\operatorname{Spin}(9,1) \times \operatorname{Spin}(1,9)$ bispinor. Similarly the bosonic equations of motion can be written as

$$
R_{a \bar{b}}+\frac{1}{16} \Phi^{-1}\left\langle F, \Gamma_{a \bar{b}} F\right\rangle=0, \quad S=0, \quad \Gamma^{A} D_{A} F=0,
$$

where $R$ and $S$ are generalised curvature tensors constructed from $D$ and $\Gamma^{A}$ are the $\operatorname{Spin}(10,10)$ gamma matrices. (The structure of the quadratic RR field term and $\Phi$ are explained in the main text.) The fermionic equations of motion are

$$
\begin{array}{ll}
\gamma^{b} D_{b} \psi_{\bar{a}}^{+}-D_{\bar{a}} \rho^{+}=\frac{1}{16} \gamma^{b} F_{\#} \gamma_{\bar{a}} \psi_{b}^{-}, & \gamma^{a} D_{a} \rho^{+}-D^{\bar{a}} \psi_{\bar{a}}^{+}=-\frac{1}{16} F_{\#} \rho^{-}, \\
\gamma^{\bar{b}} D_{\bar{b}} \psi_{a}^{-}-D_{a} \rho^{-}=\frac{1}{16} \gamma^{\bar{b}} F_{\#}^{T} \gamma_{a} \psi_{\bar{b}}^{+}, & \gamma^{\bar{a}} D_{\bar{a}} \rho^{-}-D^{a} \psi_{a}^{-}=-\frac{1}{16} F_{\#}^{T} \rho^{+} .
\end{array}
$$

and there are similar covariant expressions for the bosonic supersymmetry variations and the action.

The idea that supergravity can be reformulated with larger local symmetry groups and with a structure reflecting the duality symmetries of string theory is not new and there are several precursors of the work reported here and significant related formulations. In the mid-80s, de Wit and Nicolai considered larger structures and local symmetries related to U-duality groups in the context of eleven-dimensional supergravity in [11-13] and the generalised geometry discussed here can be viewed as the geometrical basis for their formalism in the type II context. (In [8] we will directly address the case of local $S U(8)$ symmetry considered in their original work.) Focusing on the NSNS sector, in $[14,15]$ Siegel introduced a doubled $2 d$-dimensional tangent space (on a doubled, though restricted, spacetime) with a local $G L(d, \mathbb{R}) \times G L(d, \mathbb{R})$ symmetry. He further introduced connections and curvatures, essentially defining the $R$ and $S$ curvatures that appear in (1.2).

More recently, Hull and Zwiebach introduced "double field theory" [16], motivated by $2 d$-dimensional target space models of non-geometrical backgrounds [17]. In this and subsequent work [18-21], the NSNS action is formulated in terms of first-order derivatives of doubled objects and from this equations of motion are obtained. The relation to Siegel's formalism was made in [20] and then expanded on in [22]. A closely related construction in terms of curvatures of "semi-covariant" derivatives on the doubled space was given in [23]. The result that the $\mathrm{RR}$ action can be rewritten in terms of $\operatorname{Spin}(d, d)$ spinors goes back to $[24,25]$. This and the $\operatorname{Spin}(d, d)$ formulation of the $\mathrm{RR}$ equations of motion were both considered in the double field theory formalism by Hull, who, in addition, studied the $\operatorname{Spin}(9,1) \times \operatorname{Spin}(1,9)$ field representations used in this paper from the doubled world-sheet perspective $[26]^{1}$.

\footnotetext{
${ }^{1}$ While completing this paper, we also received [50] which extends the double field theory formalism to include the RR fields and has some overlap with the results here.
} 
In earlier work, West conjectured that a non-linear realisation of $E_{11}$ underlies $\mathrm{M}$ theory [27]. In the context of type II supergravity [28], the same first-order NSNS action was recently derived in [29] and then extended to describe the RR equations of motion in [30]. Note that similar actions relevant to restrictions of eleven-dimensional supergravity to lower-dimensional manifolds are also discussed in [31] and there are further related works in [32-35].

Siegel's formalism and that of double field theory start with fields depending on $2 d$ coordinates but then impose a constraint that means, on a given coordinate patch, all the fields are independent of half the coordinates (as does West's approach). Thus locally the starting point for these formalisms is the same as that of generalised geometry. In particular, the definition of the generalised connection $D$ given here can be directly applied to the double field theory formalism. We will comment briefly on these relationships in the conclusions.

In the mathematics literature, the basic notion of the generalised tangent space with an $O(d, d)$ metric and a suitable bracket is known as an exact Courant algebroid (see $[3,36]$ and references therein). Additional "generalised geometry" structures on such objects, specifically generalised complex structures and $O(d) \times O(d)$ generalised metrics, were introduced by Hitchin and Gualtieri $[1,2]$. Connections on Courant algebroids were introduced in [37] (see also [36]) and again in [38] and [39], together with a notion of torsion and compatibility with the generalised metric.

The paper is arranged as follows. Section 2 summarises type IIA and IIB supergravity in the democratic formalism [40], using slightly modified notation, a new linear combination of dilatini and gravitini fields, and a significant rewriting of the fermionic terms. We also discuss the patching of the NSNS $B$-field and the symmetry algebra of the NSNS sector, both of which are reflected in the generalised geometry. Sections 3 and 4 introduce the key concepts of generalised geometry that we will use and show that one can always construct a torsion-free, $O(p, q) \times O(q, p)$-compatible generalised connection $D$, the analogue of the Levi-Civita connection in Riemannian geometry. In section 5 we then rewrite the type II action, supersymmetry variations and equations of motion using these new geometrical constructions. We conclude with some summary and discussion in section 6 .

\section{Type II supergravity}

Let us briefly recall the structure of $d=10$ type II supergravity. We essentially follow the conventions of the democratic formalism [40], as summarised in appendix A, and consider only the leading-order fermionic terms. We introduce a slightly unconventional notation in a few places in order to match more naturally with the underlying 
generalised geometry. It is also helpful to considerably rewrite the fermionic sector, introducing a particular linear combination of dilatini and gravitini, to match more closely what follows.

\subsection{Degrees of freedom, equations of motion and supersymmetry}

The type II fields are denoted

$$
\left\{g_{\mu \nu}, B_{\mu \nu}, \phi, A_{\mu_{1} \ldots \mu_{n}}^{(n)}, \psi_{\mu}^{ \pm}, \lambda^{ \pm}\right\}
$$

where $g_{\mu \nu}$ is the metric, $B_{\mu \nu}$ the two-form potential, $\phi$ is the dilaton and $A_{\mu_{1} \ldots \mu_{n}}^{(n)}$ are the RR potentials in the democratic formalism, with $n$ odd for type IIA and $n$ even for type IIB. In each theory there is also a pair of chiral gravitini $\psi_{\mu}^{ \pm}$and a pair of chiral dilatini $\lambda^{ \pm}$. Here our notation is that \pm does not refer to the chirality of the spinor but, as we will see, denote generalised geometrical subspaces. Specifically, in the notation of [40], for type IIA they are the chiral components of the gravitino and dilatino

$$
\begin{array}{rlrl}
\psi_{\mu} & =\psi_{\mu}^{+}+\psi_{\mu}^{-} \quad \text { where } & \gamma^{(10)} \psi_{\mu}^{ \pm}=\mp \psi_{\mu}^{ \pm} \\
\lambda & =\lambda^{+}+\lambda^{-} & \text {where } & \gamma^{(10)} \lambda^{ \pm}= \pm \lambda^{ \pm}
\end{array}
$$

(Note that $\psi_{\mu}^{+}$and $\lambda^{+}$, and similarly $\psi_{\mu}^{-}$and $\lambda^{-}$, have opposite chiralities.) For type IIB, in the notation of [40] one has two component objects

$$
\begin{aligned}
\psi_{\mu}=\left(\begin{array}{c}
\psi_{\mu}^{+} \\
\psi_{\mu}^{-}
\end{array}\right) \quad \text { where } \gamma^{(10)} \psi_{\mu}^{ \pm}=\psi_{\mu}^{ \pm} \\
\lambda=\left(\begin{array}{c}
\lambda^{+} \\
\lambda^{-}
\end{array}\right) \quad \text { where } \quad \gamma^{(10)} \lambda^{ \pm}=-\lambda^{ \pm} .
\end{aligned}
$$

and again the gravitini and dilatini have opposite chiralities.

In what follows, it will be very useful to consider the quantities

$$
\rho^{ \pm}:=\gamma^{\mu} \psi_{\mu}^{ \pm}-\lambda^{ \pm}
$$

instead of $\lambda^{ \pm}$. These are the natural combinations that appear in generalised geometry and from now on we will use $\rho^{ \pm}$rather than $\lambda^{ \pm}$.

The bosonic "pseudo-action" takes the form

$$
S_{\mathrm{B}}=\frac{1}{2 \kappa^{2}} \int \sqrt{-g}\left[\mathrm{e}^{-2 \phi}\left(\mathcal{R}+4(\partial \phi)^{2}-\frac{1}{12} H^{2}\right)-\frac{1}{4} \sum_{n} \frac{1}{n !}\left(F_{(n)}^{(B)}\right)^{2}\right],
$$


where $H=\mathrm{d} B$ and $F_{(n)}^{(B)}$ is the $n$-form RR field strength. Here we will use the " $A$-basis", where the field strengths, as sums of even or odd forms, take the form ${ }^{2}$

$$
F^{(B)}=\sum_{n} F_{(n)}^{(B)}=\sum_{n} \mathrm{e}^{B} \wedge \mathrm{d} A_{(n-1)},
$$

where $\mathrm{e}^{B}=1+B+\frac{1}{2} B \wedge B+\ldots$ This is a "pseudo-action" because the RR fields satisfy a self-duality relation that does not follow from varying the action, namely,

$$
F_{(n)}^{(B)}=(-)^{[n / 2]} * F_{(10-n)}^{(B)},
$$

where $[n]$ denotes the integer part and $* \omega$ denotes the Hodge dual of $\omega$. The fermionic action, keeping only terms quadratic in the fermions, can be written after some manipulation as

$$
\begin{aligned}
S_{\mathrm{F}}=-\frac{1}{2 \kappa^{2}} \int \sqrt{-g}\left[\mathrm { e } ^ { - 2 \phi } \left(2 \bar{\psi}^{+\mu} \gamma^{\nu} \nabla_{\nu} \psi_{\mu}^{+}-4 \bar{\psi}^{+\mu} \nabla_{\mu} \rho^{+}-2 \bar{\rho}^{+} \not \rho^{+}\right.\right. \\
\left.\quad-\frac{1}{2} \bar{\psi}^{+\mu} \not H \psi_{\mu}^{+}-\bar{\psi}_{\mu}^{+} H^{\mu \nu \lambda} \gamma_{\nu} \psi_{\lambda}^{+}-\frac{1}{2} \rho^{+} H^{\mu \nu \lambda} \gamma_{\mu \nu} \psi_{\lambda}^{+}+\frac{1}{2} \rho^{+} \not H \rho^{+}\right) \\
+\mathrm{e}^{-2 \phi}\left(2 \bar{\psi}^{-\mu} \gamma^{\nu} \nabla_{\nu} \psi_{\mu}^{-}-4 \bar{\psi}^{-\mu} \nabla_{\mu} \rho^{-}-2 \bar{\rho}^{-} \not \rho^{-}\right. \\
\left.\quad+\frac{1}{2} \bar{\psi}^{-\mu} \not H \psi_{\mu}^{-}+\bar{\psi}_{\mu}^{-} H^{\mu \nu \lambda} \gamma_{\nu} \psi_{\lambda}^{-}+\frac{1}{2} \rho^{-} H^{\mu \nu \lambda} \gamma_{\mu \nu} \psi_{\lambda}^{-}-\frac{1}{2} \rho^{-} \not H \rho^{-}\right) \\
\left.-\frac{1}{4} \mathrm{e}^{-\phi}\left(\bar{\psi}_{\mu}^{+} \gamma^{\nu} \not^{(B)} \gamma^{\mu} \psi_{\nu}^{-}+\rho^{+} \not^{(B)} \rho^{-}\right)\right] .
\end{aligned}
$$

where $\nabla$ is the Levi-Civita connection.

To match what follows it is useful to rewrite the standard equations of motion in a particular form. For the bosonic fields, with the fermions set to zero, one takes the combinations that naturally arise from the string $\beta$-functions, namely

$$
\begin{aligned}
\mathcal{R}_{\mu \nu}-\frac{1}{4} H_{\mu \lambda \rho} H_{\nu}{ }^{\lambda \rho}+2 \nabla_{\mu} \nabla_{\nu} \phi-\frac{1}{4} \mathrm{e}^{2 \phi} \sum_{n} \frac{1}{(n-1) !} F_{\mu \lambda_{1} \ldots \lambda_{n-1}}^{(B)} F_{\nu}^{(B) \lambda_{1} \ldots \lambda_{n-1}} & =0, \\
\nabla^{\mu}\left(\mathrm{e}^{-2 \phi} H_{\mu \nu \lambda}\right)-\frac{1}{2} \sum_{n} \frac{1}{(n-2) !} F_{\mu \nu \lambda_{1} \ldots \lambda_{n-2}}^{(B)} F^{(B) \lambda_{1} \ldots \lambda_{n-2}} & =0, \\
\nabla^{2} \phi-(\nabla \phi)^{2}+\frac{1}{4} \mathcal{R}-\frac{1}{48} H^{2} & =0, \\
\mathrm{~d} F^{(B)}-H \wedge F^{(B)} & =0,
\end{aligned}
$$

\footnotetext{
${ }^{2}$ Note that in type IIA one cannot write a potential for the zero-form field strength, which must instead be added by hand in (2.6). Note also that in [40] these field strengths are denoted $G$.
} 
where the final Bianchi identity for $F$ follows from the definition (2.6). Keeping only terms linear in the fermions, the fermionic equations of motion read

$$
\begin{gathered}
\gamma^{\nu}\left[\left(\nabla_{\nu} \mp \frac{1}{24} H_{\nu \lambda \rho} \gamma^{\lambda \rho}-\partial_{\nu} \phi\right) \psi_{\mu}^{ \pm} \pm \frac{1}{2} H_{\nu \mu}{ }^{\lambda} \psi_{\lambda}^{ \pm}\right]-\left(\nabla_{\mu} \mp \frac{1}{8} H_{\mu \nu \lambda} \gamma^{\nu \lambda}\right) \rho^{ \pm} \\
=\frac{1}{16} \mathrm{e}^{\phi} \sum_{n}( \pm)^{[(n+1) / 2]} \gamma^{\nu} \mathscr{F}_{(n)}^{(B)} \gamma_{\mu} \psi_{\nu}^{\mp}, \\
\left(\nabla_{\mu} \mp \frac{1}{8} H_{\mu \nu \lambda} \gamma^{\nu \lambda}-2 \partial_{\mu} \phi\right) \psi^{\mu \pm}-\gamma^{\mu}\left(\nabla_{\mu} \mp \frac{1}{24} H_{\mu \nu \lambda} \gamma^{\nu \lambda}-\partial_{\mu} \phi\right) \rho^{ \pm} \\
=\frac{1}{16} \mathrm{e}^{\phi} \sum_{n}( \pm)^{[(n+1) / 2]} \mathscr{F}_{(n)}^{(B)} \rho^{\mp},
\end{gathered}
$$

The supersymmetry variations are parametrised by are pair of chiral spinors $\epsilon^{ \pm}$ where, again, in the notation of [40], for type IIA, we have

$$
\epsilon=\epsilon^{+}+\epsilon^{-} \quad \text { where } \gamma^{(10)} \epsilon^{ \pm}=\mp \epsilon^{ \pm},
$$

while for type IIB we have the doublet

$$
\epsilon=\left(\begin{array}{l}
\epsilon^{+} \\
\epsilon^{-}
\end{array}\right) \quad \text { where } \quad \gamma^{(10)} \epsilon^{ \pm}=\epsilon^{ \pm} .
$$

Again keeping only linear terms in the fermions field, the supersymmetry transformations for the bosons read

$$
\begin{aligned}
& \delta e_{\mu}^{a}=\bar{\epsilon}^{+} \gamma^{a} \psi_{\mu}^{+}+\bar{\epsilon}^{-} \gamma^{a} \psi_{\mu}^{-}, \\
& \delta B_{\mu \nu}=2 \bar{\epsilon}^{+} \gamma_{[\mu} \psi_{\nu]}^{+}-2 \bar{\epsilon}^{-} \gamma_{[\mu} \psi_{\nu]}^{-}, \\
& \delta \phi-\frac{1}{4} \delta \log (-g)=-\frac{1}{2} \bar{\epsilon}^{+} \rho^{+}-\frac{1}{2} \bar{\epsilon}^{-} \rho^{-} \\
&\left(\mathrm{e}^{B} \wedge \delta A\right)_{\mu_{1} \ldots \mu_{n}}^{(n)}= \frac{1}{2}\left(\mathrm{e}^{-\phi} \bar{\psi}_{\nu}^{+} \gamma_{\mu_{1} \ldots \mu_{n}} \gamma^{\nu} \epsilon^{-}-\mathrm{e}^{-\phi} \bar{\epsilon}^{+} \gamma_{\mu_{1} \ldots \mu_{n}} \rho^{-}\right) \\
& \mp \frac{1}{2}\left(\mathrm{e}^{-\phi} \bar{\epsilon}^{+} \gamma^{\nu} \gamma_{\mu_{1} \ldots \mu_{n}} \psi_{\nu}^{-}+\mathrm{e}^{-\phi} \bar{\rho}^{+} \gamma_{\mu_{1} \ldots \mu_{n}} \epsilon^{-}\right),
\end{aligned}
$$

where $e_{\mu}$ is an orthonormal frame for $g_{\mu \nu}$ and in the last equation the upper sign refers to type IIA and the lower to type IIB. For the fermions one has

$$
\begin{aligned}
\delta \psi_{\mu}^{ \pm} & =\left(\nabla_{\mu} \mp \frac{1}{8} H_{\mu \nu \lambda} \gamma^{\nu \lambda}\right) \epsilon^{ \pm}+\frac{1}{16} \mathrm{e}^{\phi} \sum_{n}( \pm)^{[(n+1) / 2]} \mathscr{F}_{(n)}^{(B)} \gamma_{\mu} \epsilon^{\mp}, \\
\delta \rho^{ \pm} & =\gamma^{\mu}\left(\nabla_{\mu} \mp \frac{1}{24} H_{\mu \nu \lambda} \gamma^{\nu \lambda}-\partial_{\mu} \phi\right) \epsilon^{ \pm} .
\end{aligned}
$$

\subsection{Bosonic symmetries}

It is useful to recall the symmetries of the NSNS bosonic sector since these will be reflected in the generalised geometry. The potential $B$ is only locally defined, so that, given an open cover $\left\{U_{i}\right\}$, across coordinate patches $U_{i} \cap U_{j}$ it can be patched via

$$
B_{(i)}=B_{(j)}-\mathrm{d} \Lambda_{(i j)} .
$$


Furthermore the one-forms $\Lambda_{(i j)}$ satisfy

$$
\Lambda_{(i j)}+\Lambda_{(j k)}+\Lambda_{(k i)}=\mathrm{d} \Lambda_{(i j k)},
$$

on $U_{i} \cap U_{j} \cap U_{k}$. This makes $B$ a "connection structure on a gerbe" [41] $]^{3}$ There is a similar patching for the sum of the RR potentials $A$. We are using the "A-basis", so, given the field strengths (2.6) are globally defined we have, as a sum of forms ${ }^{4}$,

$$
A_{(i)}=\mathrm{e}^{\mathrm{d} \Lambda_{(i j)}} \wedge A_{(j)}+\mathrm{d} \hat{\Lambda}_{(i j)}
$$

where $\hat{\Lambda}_{(i j)}$ is a sum of even or odd forms in type IIA and type IIB respectively.

Focusing on the NSNS sector symmetry algebra we see that, in addition to diffeomorphism invariance, we have the local bosonic gauge symmetry

$$
B_{(i)}^{\prime}=B_{(i)}-\mathrm{d} \lambda_{(i)}, \quad A_{(i)}^{\prime}=\mathrm{e}^{\mathrm{d} \lambda_{(i)}} A_{(i)},
$$

where the choice of sign in the gauge transformation is to match the generalised geometry conventions that follow. Given the patching of $B$, the only requirement is $\mathrm{d} \lambda_{(i)}=\mathrm{d} \lambda_{(j)}$ on $U_{i} \cap U_{j}$. Thus globally $\lambda_{(i)}$ is equivalent to specifying a closed twoform. The set of gauge symmetries is then the Abelian group of closed two-forms under addition $\Omega_{\mathrm{cl}}^{2}(M)$. The gauge transformations do not commute with the diffeomorphisms so the NSNS bosonic symmetry group $G_{\text {NS }}$ has a fibred structure

$$
\Omega_{\mathrm{cl}}^{2}(M) \longrightarrow G_{\mathrm{NS}} \longrightarrow \operatorname{Diff}(M),
$$

sometimes written as the semi-direct product $\operatorname{Diff}(M) \ltimes \Omega_{\mathrm{cl}}^{2}(M)$.

One can see this structure infinitesimally by combining the diffeomorphism and gauge symmetries, given a vector $v$ and one-form $\lambda_{(i)}$, into a general variation

$$
\delta_{v+\lambda} g=\mathcal{L}_{v} g, \quad \delta_{v+\lambda} \phi=\mathcal{L}_{v} \phi, \quad \delta_{v+\lambda} B_{(i)}=\mathcal{L}_{v} B_{(i)}-\mathrm{d} \lambda_{(i)},
$$

where the patching (2.15) of $B$ implies that

$$
\mathrm{d} \lambda_{(i)}=\mathrm{d} \lambda_{(j)}-\mathcal{L}_{v} \mathrm{~d} \Lambda_{(i j)} .
$$

Recall that $\lambda_{(i)}$ and $\lambda_{(i)}+\mathrm{d} \phi_{(i)}$ define the same gauge transformation. One can use this ambiguity to integrate $(2.21)$ and set

$$
\lambda_{(i)}=\lambda_{(j)}-i_{v} \mathrm{~d} \Lambda_{(i j)}
$$

on $U_{i} \cap U_{j}$.

\footnotetext{
${ }^{3}$ In supergravity, there is no requirement that the flux $H$ is quantised. However, string theory implies the cohomological condition $H /\left(8 \pi^{2} \alpha^{\prime}\right) \in H^{3}(M, \mathbb{Z})$ (up to torsion terms). This can be implemented in the gerbe structure by requiring $g_{i j k}=\exp \left(4 \pi \alpha^{\prime} \mathrm{i} \Lambda_{(i j k)}\right)$ satisfy the cocycle condition $g_{j k l} g_{i k l}^{-1} g_{i j l} g_{i j k}^{-1}=1$ on $U_{i} \cap U_{j} \cap U_{k} \cap U_{l}$. We will not consider this further restriction in the following.

${ }^{4}$ Note here $i$ and $j$ refer to the patch not the degree of the form.
} 


\section{$3 \quad O(d, d) \times \mathbb{R}^{+}$generalised geometry}

We would like to define the generalised geometric analogues of each of the ingredients in the construction of the Levi-Civita connection ${ }^{5}$. In this section we review the generalisations of the frame bundle, the Lie derivative, connections, torsion and curvature. In the following section we discuss the notion of a generalised metric and the analogue of the Levi-Civita connection.

One way to view generalised geometry is as a formalism for "geometrising" the bosonic structures that appear in supergravity. In the context of the NSNS sector this means first combining the symmetry algebra of diffeomorphisms and $B$-field gauge transformations into an algebra of "generalised" Lie derivatives. This structure is known as an "exact Courant algebroid" in the mathematics literature $[3,36]$ and, on a $d$-dimensional manifold, defines a bundle with a natural $O(d, d)$ action. Combining $g, B$ and $\phi$ into a single geometrical object introduces an additional refinement of the structure, defining a generalised geometry $[1,2]$. The only slight, though important, extension we will require here is to promote the $O(d, d)$ action to $O(d, d) \times \mathbb{R}^{+}[42,43]$.

\subsection{Generalised structure bundle}

We start by recalling the generalised tangent space and defining what we will call the "generalised structure" which is the analogue of the frame bundle $F$ in conventional geometry.

Let $M$ be a $d$-dimensional spin manifold. In line with the patching of the transformation parameters (2.22), one starts by defining the generalised tangent space $E$. It is defined as an extension of the tangent space by the cotangent space

$$
0 \longrightarrow T^{*} M \longrightarrow E \longrightarrow T M \longrightarrow 0,
$$

which depends on the patching one-forms $\Lambda_{(i j)}$. If $v_{(i)} \in \Gamma\left(T U_{i}\right)$ and $\lambda_{(i)} \in \Gamma\left(T^{*} U_{i}\right)$, so $V_{(i)}=v_{(i)}+\lambda_{(i)}$ is a section of $E$ over the patch $U_{i}$, then

$$
v_{(i)}+\lambda_{(i)}=v_{(j)}+\left(\lambda_{(j)}-i_{v_{(j)}} \mathrm{d} \Lambda_{(i j)}\right),
$$

on the overlap $U_{i} \cap U_{j}$. Hence as defined, while the $v_{(i)}$ globally are equivalent to a choice of vector, the $\lambda_{(i)}$ do not globally define a one-form. $E$ is in fact isomorphic to $T M \oplus T^{*} M$ though there is no canonical isomorphism. Instead one must choose a splitting of the sequence (3.1) as discussed below. Crucially the definition of $E$ is consistent with an $O(d, d)$ metric given by, for $V=v+\lambda$

$$
\langle V, V\rangle=i_{v} \lambda,
$$

\footnotetext{
${ }^{5}$ These ingredients are reviewed in appendix B.
} 
since $i_{v_{(i)}} \lambda_{(i)}=i_{v_{(j)}} \lambda_{(j)}$ on $U_{i} \cap U_{j}$.

In order to describe the dilaton correctly we will actually need to consider a slight generalisation of $E$. We define the bundle $\tilde{E}$ weighted by $\operatorname{det} T^{*} M$ so that

$$
\tilde{E}=\operatorname{det} T^{*} M \otimes E .
$$

The point is that, given the metric (3.3), one can now define a natural principal bundle with fibre $O(d, d) \times \mathbb{R}^{+}$in terms of bases of $\tilde{E}$. We define a conformal basis $\left\{\hat{E}_{A}\right\}$ with $A=1, \ldots 2 d$ on $\tilde{E}_{x}$ as one satisfying

$$
\left\langle\hat{E}_{A}, \hat{E}_{B}\right\rangle=\Phi^{2} \eta_{A B} \quad \text { where } \quad \eta=\frac{1}{2}\left(\begin{array}{ll}
0 & 1 \\
1 & 0
\end{array}\right) .
$$

That is $\left\{\hat{E}_{A}\right\}$ is orthonormal up to a frame-dependent conformal factor $\Phi \in \Gamma\left(\operatorname{det} T^{*} M\right)$. We then define the generalised structure bundle

$$
\tilde{F}=\left\{\left(x,\left\{\hat{E}_{A}\right\}\right): x \in M \text {, and }\left\{\hat{E}_{A}\right\} \text { is a conformal basis of } \tilde{E}_{x}\right\} .
$$

By construction, this is a principal bundle with fibre $O(d, d) \times \mathbb{R}^{+}$. One can make a change of basis

$$
V^{A} \mapsto V^{\prime A}=M_{B}^{A} V^{B}, \quad \hat{E}_{A} \mapsto \hat{E}_{A}^{\prime}=\hat{E}_{B}\left(M^{-1}\right)^{B}{ }_{A} .
$$

where $M \in O(d, d) \times \mathbb{R}^{+}$so that $\left(M^{-1}\right)^{C}{ }_{A}\left(M^{-1}\right)^{D}{ }_{B} \eta_{C D}=\sigma^{2} \eta_{A B}$ for some $\sigma$. The topology of $\tilde{F}$ encodes both the topology of the tangent bundle $T M$ and of the $B$-field gerbe.

Given the definition (3.1) there is one natural conformal basis defined by the choice of coordinates on $M$, namely $\left\{\hat{E}_{A}\right\}=\left\{\partial / \partial x^{\mu}\right\} \cup\left\{\mathrm{d} x^{\mu}\right\}$. Given $V \in \Gamma(E)$ over the patch $U_{i}$, we have $V=v^{\mu}\left(\partial / \partial x^{\mu}\right)+\lambda_{\mu} \mathrm{d} x^{\mu}$, we will sometime denote the components of $V$ in this frame by an index $M$ such that

$$
V^{M}=\left\{\begin{array}{ll}
v^{\mu} & \text { for } M=\mu \\
\lambda_{\mu} & \text { for } M=\mu+d
\end{array} .\right.
$$

\subsection{Generalised tensors and spinors and split frames}

Generalised tensors are simply sections of vector bundles constructed from different representations of $O(d, d) \times \mathbb{R}^{+}$, that is representations of $O(d, d)$ of definite weight under $\mathbb{R}^{+}$. Since the $O(d, d)$ metric gives an isomorphism between $E$ and $E^{*}$, one has the bundle

$$
E_{(p)}^{\otimes n}=\left(\operatorname{det} T^{*} M\right)^{p} \otimes E \otimes \cdots \otimes E
$$


for a general tensor of weight $p$.

One can also consider $\operatorname{Spin}(d, d)$ spinor representations [2]. The $O(d, d)$ Clifford algebra

$$
\left\{\Gamma_{A}, \Gamma_{B}\right\}=2 \eta_{A B}
$$

can be realised on each coordinate patch $U_{i}$ by identifying spinors with weighted sums of forms $\Psi_{(i)} \in \Gamma\left(\left(\operatorname{det} T^{*} U_{i}\right)^{1 / 2} \otimes \Lambda^{\bullet} T^{*} U_{i}\right)$, with the Clifford action

$$
V^{A} \Gamma_{A} \Psi_{(i)}=i_{v} \Psi_{(i)}+\lambda_{(i)} \wedge \Psi_{(i)} .
$$

The patching (3.2) then implies

$$
\Psi_{(i)}=\mathrm{e}^{\mathrm{d} \Lambda_{(i j)}} \wedge \Psi_{(j)} .
$$

Projecting onto the chiral spinors then defines two $\operatorname{Spin}(d, d)$ spinor bundles, isomorphic to weighted sums of odd or even forms $S^{ \pm}(E) \simeq\left(\operatorname{det} T^{*} M\right)^{-1 / 2} \otimes \Lambda^{\text {even/odd }} T^{*} M$ where again specifying the isomorphism requires a choice of splitting.

More generally one defines $\operatorname{Spin}(d, d) \times \mathbb{R}^{+}$spinors of weight $p$ as sections of

$$
S_{(p)}^{ \pm}=\left(\operatorname{det} T^{*} M\right)^{p} \otimes S^{ \pm}(E) .
$$

Note that there is a natural $\operatorname{Spin}(d, d)$ invariant bilinear on these spinor spaces given by the Mukai pairing $[1,2]$. For $\Psi, \Psi^{\prime} \in \Gamma\left(S_{(p)}^{ \pm}\right)$one has

$$
\left\langle\Psi, \Psi^{\prime}\right\rangle=\sum_{n}(-)^{[(n+1) / 2]} \Psi^{(d-n)} \wedge \Psi^{\prime(n)} \in \Gamma\left(\left(\operatorname{det} T^{*} M\right)^{2 p}\right)
$$

where $\Psi^{(n)}$ and $\Psi^{\prime(n)}$ are the local weighted $n$-form components.

A special class of conformal frames are those defined by a splitting of the generalised tangent space $E$. A splitting is a map $T M \rightarrow E$. It is equivalent to specifying a local two-form $B$ patched as in (2.15) and defines an isomorphism $E \simeq T M \oplus T^{*} M$. If $\left\{\hat{e}_{a}\right\}$ is a generic basis for $T M$ and $\left\{e^{a}\right\}$ be the dual basis on $T^{*} M$, one can then define what we call a split frame $\left\{\hat{E}_{A}\right\}$ for $\tilde{E}$ by

$$
\hat{E}_{A}=\left\{\begin{array}{ll}
\hat{E}_{a}=(\operatorname{det} e)\left(\hat{e}_{a}+i_{\hat{e}_{a}} B\right) & \text { for } A=a \\
E^{a}=(\operatorname{det} e) e^{a} & \text { for } A=a+d
\end{array} .\right.
$$

We immediately see that

$$
\left\langle\hat{E}_{A}, \hat{E}_{B}\right\rangle=(\operatorname{det} e)^{2} \eta_{A B}
$$

and hence the basis is conformal. Writing $V=v^{a} \hat{E}_{a}+\lambda_{a} E^{a} \in \Gamma(\tilde{E})$ we have

$$
\begin{aligned}
V^{(B)} & =v^{a}(\operatorname{det} e) \hat{e}_{a}+\lambda_{a}(\operatorname{det} e) e^{a} \\
& =v_{(i)}+\lambda_{(i)}-i_{v_{(i)}} B_{(i)},
\end{aligned}
$$


demonstrating that the splitting defines an isomorphism $\tilde{E} \simeq\left(\operatorname{det} T^{*} M\right) \otimes\left(T M \oplus T^{*} M\right)$ since $\lambda_{(i)}-i_{v_{(i)}} B_{(i)}=\lambda_{(j)}-i_{v_{(j)}} B_{(j)}$.

The class of split frames defines a sub-bundle of $\tilde{F}$. Such frames are related by transformations (3.7) where $M$ takes the form

$$
M=(\operatorname{det} A)^{-1}\left(\begin{array}{ll}
1 & 0 \\
\omega & 1
\end{array}\right)\left(\begin{array}{cc}
A & 0 \\
0 & \left(A^{-1}\right)^{T}
\end{array}\right)
$$

where $A \in G L(d, \mathbb{R})$ is the matrix transforming $\hat{e}_{a} \mapsto \hat{e}_{b}\left(A^{-1}\right)^{b}{ }_{a}$ while $\omega=\frac{1}{2} \omega_{a b} e^{a} \wedge e^{b}$ transforms $B \mapsto B^{\prime}=B+\omega$, where $\omega$ must be closed for $B^{\prime}$ to be a splitting. This defines a parabolic subgroup $G_{\text {split }}=G L(d, \mathbb{R}) \ltimes \mathbb{R}^{d(d-1) / 2} \subset O(d, d) \times \mathbb{R}^{+}$and hence the set of all frames of the form (3.15) defines a $G_{\text {split }}$ principal sub-bundle of $\tilde{F}$, that

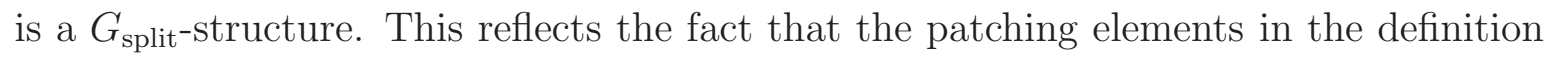
of $\tilde{E}$ lie only in this subgroup of $O(d, d) \times \mathbb{R}^{+}$.

In what follows it will be useful to also define a class of conformal split frames given by the set of split bases conformally rescaled by a function $\phi$ so that

$$
\hat{E}_{A}=\left\{\begin{array}{ll}
\hat{E}_{a}=\mathrm{e}^{-2 \phi}(\operatorname{det} e)\left(\hat{e}_{a}+i_{\hat{e}_{a}} B\right) & \text { for } A=a \\
E^{a}=\mathrm{e}^{-2 \phi}(\operatorname{det} e) e^{a} & \text { for } A=a+d
\end{array} .\right.
$$

thus defining a $G_{\text {split }} \times \mathbb{R}^{+}$sub-bundle of $\tilde{F}$. In complete analogy with the split case, the components of $V \in \Gamma(\tilde{E})$ in the conformally split frame are related to those in the coordinate basis by

$$
V^{(B, \phi)}=\mathrm{e}^{2 \phi}\left(v_{(i)}+\lambda_{(i)}-i_{v_{(i)}} B_{(i)}\right) .
$$

We can similarly write the components of generalised spinors in different frames. The relation between the coordinate and split frames implies that if $\Psi_{a_{1} \ldots a_{n}}^{(B)}$ are the polyform components of $\Psi \in \Gamma\left(S_{(p)}^{ \pm}\right)$in the split frame then

$$
\Psi^{(B)}=\sum_{n} \frac{1}{n !} \Psi_{a_{1} \ldots a_{n}}^{(B)} e^{a_{1}} \wedge \cdots \wedge e^{a_{n}}=\mathrm{e}^{B_{(i)}} \wedge \Psi_{(i)}
$$

demonstrating the isomorphism $S_{(p)}^{ \pm} \simeq\left(\operatorname{det} T^{*} M\right)^{p-1 / 2} \otimes \Lambda^{\text {even/odd }} T^{*} M$, since $\mathrm{e}^{B_{(i)}} \wedge$ $\Psi_{(i)}=\mathrm{e}^{B_{(j)}} \wedge \Psi_{(j)}$. In the conformal split frame one similarly has

$$
\Psi^{(B, \phi)}=\mathrm{e}^{p \phi} \mathrm{e}^{B_{(i)}} \wedge \Psi_{(i)}
$$

\subsection{The Dorfman derivative, Courant bracket and exterior derivative}

An important property of the generalised tangent space is that it admits a generalisation of the Lie derivative which encodes the bosonic symmetries of the NSNS sector of type 
II supergravity. Given $V=v+\lambda \in \Gamma(E)$, one can define an operator $L_{V}$ acting on any generalised tensor, which combines the action of an infinitesimal diffeomorphisms generated by $v$ and a $B$-field gauge transformations generated by $\lambda$.

Acting on $W=w+\zeta \in E_{(p)}$, we define the Dorfman derivative 6 or "generalised Lie derivative" as [42]

$$
L_{V} W=\mathcal{L}_{v} w+\mathcal{L}_{v} \zeta-i_{w} \mathrm{~d} \lambda
$$

where, since $w$ and $\zeta$ are weighted tensors, the action of the Lie derivative is

$$
\begin{aligned}
\mathcal{L}_{v} w^{\mu} & =v^{\nu} \partial_{\nu} w^{\mu}-w^{\nu} \partial_{\nu} v^{\mu}+p\left(\partial_{\nu} v^{\nu}\right) w^{\mu} \\
\mathcal{L}_{v} \zeta_{\mu} & =v^{\nu} \partial_{\nu} \zeta_{\mu}+\left(\partial_{\mu} v^{\nu}\right) \zeta_{\nu}+p\left(\partial_{\nu} v^{\nu}\right) \zeta^{\mu}
\end{aligned}
$$

Defining the action on a function $f$ as simply $L_{V} f=\mathcal{L}_{v} f$, one can then extend the notion of Dorfman derivative to any $O(d, d) \times \mathbb{R}^{+}$tensor using the Leibniz property.

To see this explicitly it is useful to note that we can rewrite (3.23) in a more $O(d, d) \times \mathbb{R}^{+}$covariant way, in analogy with (B.4). First note that one can embed the action of the partial derivative operator into generalised geometry using the map $T^{*} M \rightarrow E$. In coordinate indices, as viewed as mapping to a section of $E^{*}$, one defines

$$
\partial_{M}=\left\{\begin{array}{ll}
\partial_{\mu} & \text { for } M=\mu \\
0 & \text { for } M=\mu+d
\end{array} .\right.
$$

One can then rewrite (3.23) in terms of generalised objects (as in $[14,15,20]$ )

$$
L_{V} W^{M}=V^{N} \partial_{N} W^{M}+\left(\partial^{M} V^{N}-\partial^{N} V^{M}\right) W_{N}+p\left(\partial_{N} V^{N}\right) W^{M},
$$

where indices are contracted using the $O(d, d)$ metric $(3.3)$, which, by definition, is constant with respect to $\partial$. Note that this form is exactly analogous to the conventional Lie derivative (B.4), though now with the adjoint action in $\mathfrak{o}(d, d) \oplus \mathbb{R}$ rather than $\mathfrak{g l}(d)$. Specifically the second and third terms are (minus) the action of an $\mathfrak{o}(d, d) \oplus \mathbb{R}$ element $m$, given by

$$
m \cdot W=\left(\begin{array}{cc}
a & 0 \\
-\omega & -a^{T}
\end{array}\right)\left(\begin{array}{l}
w \\
\zeta
\end{array}\right)-p \operatorname{tr} a\left(\begin{array}{l}
w \\
\zeta
\end{array}\right),
$$

where $a_{\nu}^{\mu}=\partial_{\nu} v^{\mu}$ and $\omega_{\mu \nu}=\partial_{\mu} \lambda_{\nu}-\partial_{\nu} \lambda_{\mu}$. Comparing with (3.18), we see that $m$ in fact acts in the Lie algebra of the $G_{\text {split }}$ subgroup of $O(d, d) \times \mathbb{R}^{+}$.

This form can then be naturally extended to an arbitrary $O(d, d) \times \mathbb{R}^{+}$tensor $\alpha \in \Gamma\left(E_{(p)}^{\otimes n}\right)$ as

$$
\begin{aligned}
L_{V} \alpha^{M_{1} \ldots M_{n}}=V^{N} \partial_{N} \alpha^{M_{1} \ldots M_{n}}+\left(\partial^{M_{1}} V^{N}-\partial^{N} V^{M_{1}}\right) \alpha_{N}{ }^{M_{2} \ldots M_{n}} \\
\\
+\cdots+\left(\partial^{M_{n}} V^{N}-\partial^{N} V^{M_{n}}\right) \alpha^{M_{1} \ldots M_{n-1}}+p\left(\partial_{N} V^{N}\right) W^{M}
\end{aligned}
$$

\footnotetext{
${ }^{6}$ If $p=0$ then $L_{V} W$ is none other than the Dorfman bracket [44]. Since it extends to a derivation on the tensor algebra of generalised tensors, it is natural in our context to call it the "Dorfman derivative".
} 
again in analogy with (B.4). It similarly extends to generalised spinors $\Psi \in \Gamma\left(S_{(p)}^{ \pm}\right)$as (see also [50])

$$
L_{V} \Psi=V^{N} \partial_{N} \Psi+\frac{1}{4}\left(\partial_{M} V_{N}-\partial_{N} V_{M}\right) \Gamma^{M N} \Psi+p\left(\partial_{M} V^{M}\right) \Psi
$$

where $\Gamma_{M N}=\frac{1}{2}\left(\Gamma_{M} \Gamma_{N}-\Gamma_{N} \Gamma_{M}\right)$.

Note that when $W \in \Gamma(E)$ one can also define the antisymmetrisation of the Dorfman derivative

$$
\begin{aligned}
\llbracket V, W \rrbracket & =\frac{1}{2}\left(L_{V} W-L_{W} V\right) \\
& =[v, w]+\mathcal{L}_{v} \zeta-\mathcal{L}_{w} \lambda-\frac{1}{2} \mathrm{~d}\left(i_{v} \zeta-i_{w} \lambda\right),
\end{aligned}
$$

which is known as the Courant bracket [45]. It can be rewritten in an $O(d, d)$ covariant form as

$$
\llbracket U, V \rrbracket^{M}=U^{N} \partial_{N} V^{M}-V^{N} \partial_{N} U^{M}-\frac{1}{2}\left(U_{N} \partial^{M} V^{N}-V_{N} \partial^{M} U^{N}\right) .
$$

which follows directly from (3.26).

Finally note that since $S_{(1 / 2)}^{ \pm} \simeq \Lambda^{\text {even/odd }} T^{*} M$ the Clifford action of $\partial_{M}$ on $\Psi \in$ $\Gamma\left(S_{(1 / 2)}^{ \pm}\right)$defines a natural action of the exterior derivative. On $U_{i}$ one defines $d$ : $\Gamma\left(S_{(1 / 2)}^{ \pm}\right) \rightarrow \Gamma\left(S_{(1 / 2)}^{\mp}\right)$ by

$$
(\mathrm{d} \Psi)_{(i)}=\frac{1}{2} \Gamma^{M} \partial_{M} \Psi_{(i)}=\mathrm{d} \Psi_{(i)}
$$

that is, it is simply the exterior derivative of the component $p$-forms. The Dorfman derivative and Courant bracket can then be regarded as derived brackets for this exterior derivative [46].

\subsection{Generalised $O(d, d) \times \mathbb{R}^{+}$connections and torsion}

We now turn to the definitions of generalised connections, torsion and the possibility of defining a generalised curvature. The notion of connection on a Courant algebroid was first introduced by Alekseev and Xu [36, 37] and Gualtieri [39] (see also Ellwood [38]). At least locally, it is also essentially equivalent to the connection defined by Siegel [14, 15] and discussed in double field theory [22]. It is also very closely related to the differential operator introduced in the "stringy differential geometry" of [23].

Our definitions will follow closely those in $[37,39]$ though, in connecting to supergravity, it is important to extend the definitions to include the $\mathbb{R}^{+}$factor in the generalised structure bundle. 


\subsubsection{Generalised connections}

Here we will specifically be interested in those generalised connections that are compatible with the $O(d, d) \times \mathbb{R}^{+}$structure. Following $[37,39]$ we can define a first-order linear differential operator $D$, such that, given $W \in \Gamma(\tilde{E})$, in frame indices,

$$
D_{M} W^{A}=\partial_{M} W^{A}+\tilde{\Omega}_{M}{ }_{B}{ }_{B} W^{B} .
$$

Compatibility with the $O(d, d) \times \mathbb{R}^{+}$structure implies

$$
\tilde{\Omega}_{M}{ }^{A}{ }_{B}=\Omega_{M}{ }^{A}{ }_{B}-\Lambda_{M} \delta^{A}{ }_{B},
$$

where $\Lambda$ is the $\mathbb{R}^{+}$part of the connection and $\Omega$ the $O(d, d)$ part, so that we have

$$
\Omega_{M}^{A B}=-\Omega_{M}{ }^{B A}
$$

The action of $D$ then extends naturally to any generalised tensor. In particular, if $\alpha \in \Gamma\left(E_{(p)}^{\otimes n}\right)$ we have

$$
\begin{aligned}
D_{M} \alpha^{A_{1} \ldots A_{n}}=\partial_{M} \alpha^{A_{1} \ldots A_{n}} & +\Omega_{M}{ }^{A_{1}}{ }_{B} \alpha^{B A_{2} \ldots A_{n}} \\
& +\cdots+\Omega_{M}{ }^{A_{n}}{ }_{B} \alpha^{A_{1} \ldots A_{n-1} B}-p \Lambda_{M} \alpha^{A_{1} \ldots A_{n}} .
\end{aligned}
$$

Similarly, if $\Psi \in \Gamma\left(S_{(p)}^{ \pm}\right)$then

$$
D_{M} \Psi=\left(\partial_{M}+\frac{1}{4} \Omega_{M}^{A B} \Gamma_{A B}-p \Lambda_{M}\right) \Psi .
$$

Given a conventional connection $\nabla$ and a conformal split frame of the form (3.19), one can construct the corresponding generalised connection as follows. Writing a generalised vector $W \in \Gamma(\tilde{E})$ as

$$
W=W^{A} \hat{E}_{A}=w^{a} \hat{E}_{a}+\zeta_{a} E^{a},
$$

by construction $w=w^{a}(\operatorname{det} e) \hat{e}_{a} \in \Gamma\left(\left(\operatorname{det} T^{*} M\right) \otimes T M\right)$ and $\zeta=\zeta_{a}(\operatorname{det} e) e^{a} \in \Gamma\left(\left(\operatorname{det} T^{*} M\right) \otimes\right.$ $\left.T^{*} M\right)$ and so we can define $\nabla_{\mu} w^{a}$ and $\nabla_{\mu} \zeta_{a}$. The generalised connection defined by $\nabla$ lifted to an action on $\tilde{E}$ by the conformal split frame is then simply

$$
\left(D_{M}^{\nabla} W^{A}\right) \hat{E}_{A}=\left\{\begin{array}{ll}
\left(\nabla_{\mu} w^{a}\right) \hat{E}_{a}+\left(\nabla_{\mu} \zeta_{a}\right) E^{a} & \text { for } M=\mu \\
0 & \text { for } M=\mu+d
\end{array} .\right.
$$




\subsubsection{Generalised torsion}

We define the generalised torsion $T$ of a generalised connection $D$ in direct analogy to the conventional definition (B.8). Let $\alpha$ be any generalised tensor and $L_{V}^{D} \alpha$ be the Dorfman derivative (3.28) with $\partial$ replaced by $D$. The generalised torsion is a linear map $T: \Gamma(E) \rightarrow \Gamma(\operatorname{ad}(\tilde{F}))$ where $\operatorname{ad}(\tilde{F}) \simeq \Lambda^{2} E \oplus \mathbb{R}$ is the $\mathfrak{o}(d, d) \oplus \mathbb{R}$ adjoint representation bundle associated to $\tilde{F}$. It is defined by

$$
T(V) \cdot \alpha=L_{V}^{D} \alpha-L_{V} \alpha,
$$

for any $V \in \Gamma(E)$ and where $T(V)$ acts via the adjoint representation on $\alpha$. This definition is close to that of [39], except for the additional $\mathbb{R}^{+}$action in the definition of $L$.

Viewed as a tensor $T \in \Gamma(E \otimes$ ad $\tilde{F})$, with indices such that $T(V)^{M}{ }_{N}=V^{P} T^{M}{ }_{P N}$, we can derive an explicit expression for $T$. Let $\left\{\hat{E}_{A}\right\}$ be a general conformal basis with $\left\langle\hat{E}_{A}, \hat{E}_{B}\right\rangle=\Phi^{2} \eta_{A B}$. Then $\left\{\Phi^{-1} \hat{E}_{A}\right\}$ is an orthonormal basis for $E$. Given the connection $D_{M} W^{A}=\partial_{M} W^{A}+\tilde{\Omega}_{M}{ }^{A}{ }_{B} W^{B}$, we have

$$
T_{A B C}=-3 \tilde{\Omega}_{[A B C]}+\tilde{\Omega}_{D}{ }^{D}{ }_{B} \eta_{A C}-\Phi^{-2}\left\langle\hat{E}_{A}, L_{\Phi^{-1} \hat{E}_{B}} \hat{E}_{C}\right\rangle,
$$

where indices are lowered with $\eta_{A B}$.

Naively one might expect that $T \in \Gamma\left(\left(E \otimes \Lambda^{2} E\right) \oplus E\right)$. However the form of the Dorfman derivative means that fewer components of $\tilde{\Omega}$ actually enter the torsion and

$$
T \in \Gamma\left(\Lambda^{3} E \oplus E\right) .
$$

This can be seen most easily in the coordinate basis where the two components are

$$
T_{P N}^{M}=\left(T_{1}\right)^{M}{ }_{P N}-\left(T_{2}\right)_{P} \delta^{M}{ }_{N},
$$

with

$$
\begin{aligned}
\left(T_{1}\right)_{M N P} & =-3 \tilde{\Omega}_{[M N P]}=-3 \Omega_{[M N P]}, \\
\left(T_{2}\right)_{M} & =-\tilde{\Omega}_{Q}{ }^{Q}{ }_{M}=\Lambda_{M}-\Omega_{Q}{ }^{Q}{ }_{M} .
\end{aligned}
$$

An immediate consequence of this definition is that for $\Psi \in \Gamma\left(S_{(1 / 2)}^{ \pm}\right)$the Dirac operator $\Gamma^{M} D_{M} \Psi$ is determined by the torsion of the connection [37]

$$
\begin{aligned}
\Gamma^{M} D_{M} \Psi & =\Gamma^{M}\left(\partial_{M} \Psi+\frac{1}{4} \Omega_{M N P} \Gamma^{N P} \Psi-\frac{1}{2} \Lambda_{M} \Psi\right) \\
& =\Gamma^{M} \partial_{M} \Psi+\frac{1}{4} \Omega_{[M N P]} \Gamma^{M N P} \Psi-\frac{1}{2}\left(\Lambda_{M}-\Omega_{N}{ }^{N}{ }_{M}\right) \Gamma^{M} \Psi \\
& =2 \mathrm{~d} \Psi-\frac{1}{12}\left(T_{1}\right)_{[M N P]} \Gamma^{M N P} \Psi-\frac{1}{2}\left(T_{2}\right)_{M} \Gamma^{M} \Psi .
\end{aligned}
$$


This equation could equally well be used as a definition of the torsion of a generalised connection. Note in particular that if the connection is torsion-free we see that the Dirac operator becomes equal to the exterior derivative

$$
\Gamma^{M} D_{M} \Psi=2 \mathrm{~d} \Psi .
$$

As an example, we can calculate the torsion for the generalised connection $D^{\nabla}$ defined in (3.39). In general we have

$$
L_{\Phi^{-1} \hat{E}_{A}} \hat{E}_{B}=\left(L_{\Phi^{-1} \hat{E}_{A}} \Phi\right) \Phi^{-1} \hat{E}_{B}+\Phi\left(L_{\Phi^{-1} \hat{E}_{A}}\left(\Phi^{-1} \hat{E}_{B}\right)\right)
$$

where here

$$
L_{\Phi^{-1} \hat{E}_{A}} \Phi= \begin{cases}-\mathrm{e}^{-2 \phi}(\operatorname{det} e)\left(i_{\hat{e}_{a}} i_{\hat{e}_{b}} \mathrm{~d} e^{b}+2 i_{\hat{e}_{a}} \mathrm{~d} \phi\right) & \text { for } A=a \\ 0 & \text { for } A=a+d\end{cases}
$$

and

$$
L_{\Phi^{-1} \hat{E}_{A}} \Phi^{-1} \hat{E}_{B}=\left(\begin{array}{cc}
{\left[\hat{e}_{a}, \hat{e}_{b}\right]+i_{\left[\hat{e}_{a}, \hat{e}_{b}\right]} B-i_{\hat{e}_{a}} i_{\hat{e}_{b}} H} & \mathcal{L}_{\hat{e}_{a}} e^{b} \\
-\mathcal{L}_{\hat{e}_{b}} e^{a} & 0
\end{array}\right)_{A B},
$$

where $H=\mathrm{d} B$. If the conventional connection $\nabla$ is torsion-free, the corresponding generalised torsion is given by

$$
T_{1}=-4 H, \quad T_{2}=-4 \mathrm{~d} \phi,
$$

where we are using the embedding ${ }^{7} T^{*} M \rightarrow E$ (and the corresponding $T^{*} M \rightarrow \Lambda^{3} E$ ) to write the expressions in terms of forms. This result is most easily seen by taking $\hat{e}_{a}$ to be the coordinate frame, so that all but the $H$ and $\mathrm{d} \phi$ terms in (3.48) and (3.49) vanish.

\subsubsection{The absence of generalised curvature}

Having defined torsion it is natural to ask if one can also introduce a notion of generalised curvature in analogy to the usual definition (B.9), as the commutator of two generalised connections but now using the Courant bracket (3.30) rather than the Lie bracket

$$
R(U, V, W)=\left[D_{U}, D_{V}\right] W-D_{\llbracket U, V \rrbracket} W .
$$

However, this object is non-tensorial [39]. We can check for linearity in the arguments explicitly. Taking $U \rightarrow f U, V \rightarrow g V$ and $W \rightarrow h W$ for some scalar functions $f, g, h$, we obtain

$$
\begin{aligned}
& {\left[D_{f U}, D_{g V}\right] h W-D_{\llbracket f U, g V \rrbracket} h W} \\
& \quad=f g h\left(\left[D_{U}, D_{V}\right] W-D_{\llbracket U, V \rrbracket} W\right)-\frac{1}{2} h\langle U, V\rangle D_{(f \mathrm{~d} g-g \mathrm{~d} f)} W
\end{aligned}
$$

\footnotetext{
${ }^{7}$ Note that with our definitions we have $\left(\partial^{A} \phi\right) \Phi^{-1} \hat{E}_{A}=2 \mathrm{~d} \phi$ due to the factor $\frac{1}{2}$ in $\eta_{A B}$
} 
and so the curvature is not linear in $U$ and $V$.

Nonetheless, if there is additional structure, as will be relevant for supergravity, we are able to define other tensorial objects that are measures of generalised curvature. In particular, let $C_{1} \subset E$ and $C_{2} \subset E$ be subspaces such that $\langle U, V\rangle=0$ for all $U \in \Gamma\left(C_{1}\right)$ and $V \in \Gamma\left(C_{2}\right)$. For such a $U$ and $V$ the final term in (3.52) vanishes, and so $R \in \Gamma\left(\left(C_{1} \otimes C_{2}\right) \otimes \mathfrak{o}(d, d)\right)$ is a tensor. A special example of this is when $C_{1}=C_{2}$ is a null subspace of $E$.

\section{$4 O(p, q) \times O(q, p)$ structures and torsion-free connections}

We now turn to constructing the generalised analogue of the Levi-Civita connection. The latter is the unique torsion-free connection that preserves the $O(d) \subset G L(d, \mathbb{R})$ structure defined by a metric $g$. Here we will be interested in generalised connections that preserve an $O(p, q) \times O(q, p) \subset O(d, d) \times \mathbb{R}^{+}$structure on $\tilde{F}$, where $p+q=d$. We will find that, in analogy to the Levi-Civita connection, it is always possible to construct torsion-free connections of this type but there is no unique choice. Locally this is same construction that appears in Siegel $[14,15]$ and closely related to that of $[23]$.

\section{1 $O(p, q) \times O(q, p)$ structures and the generalised metric}

Following closely the standard definition of the generalised metric [2], consider an $O(p, q) \times O(q, p)$ principal sub-bundle $P$ of the generalised structure bundle $\tilde{F}$. As discussed below, this is equivalent to specifying a conventional metric $g$ of signature $(p, q)$, a $B$-field patched as in (2.15) and a dilaton $\phi$. As such it clearly gives the appropriate generalised structure to capture the NSNS supergravity fields.

Geometrically, an $O(p, q) \times O(q, p)$ structure does two things. First it fixes a nowhere vanishing section $\Phi \in \Gamma\left(\operatorname{det} T^{*} M\right)$, giving an isomorphism between weighted and unweighted generalised tangent space $\tilde{E}$ and $E$. Second it defines a splitting of $E$ into two $d$-dimensional sub-bundles

$$
E=C_{+} \oplus C_{-}
$$

such that the $O(d, d)$ metric (3.3) restricts to a separate metric of signature $(p, q)$ on $C_{+}$and a metric of signature $(q, p)$ on $C_{-}$. (Each sub-bundle is also isomorphic to $T M$ using the map $E \rightarrow T M$.)

In terms of $\tilde{F}$ we can identify a special set of frames defining a $O(p, q) \times O(p, q)$

sub-bundle. We define a frame $\left\{\hat{E}_{a}^{+}\right\} \cup\left\{\hat{E}_{\bar{a}}^{-}\right\}$such that $\left\{\hat{E}_{a}^{+}\right\}$form an orthonormal 
basis for $C_{+}$and $\left\{\hat{E}_{\bar{a}}^{-}\right\}$form an orthonormal basis for $C_{-}$. This means they satisfy

$$
\begin{aligned}
& \left\langle\hat{E}_{a}^{+}, \hat{E}_{b}^{+}\right\rangle=\Phi^{2} \eta_{a b}, \\
& \left\langle\hat{E}_{\bar{a}}^{-}, \hat{E}_{\bar{b}}^{-}\right\rangle=-\Phi^{2} \eta_{\bar{a} \bar{b}}, \\
& \left\langle\hat{E}_{a}^{+}, \hat{E}_{\bar{a}}^{-}\right\rangle=0 .
\end{aligned}
$$

where $\Phi \in \Gamma\left(\operatorname{det} T^{*} M\right)$ is now some fixed density (independent of the particular frame element) and $\eta_{a b}$ and $\eta_{\bar{a} \bar{b}}$ are flat metrics with signature $(p, q)$. There is thus a manifest $O(p, q) \times O(q, p)$ symmetry with the first factor acting on $\hat{E}_{a}^{+}$and the second on $\hat{E}_{\bar{a}}^{-}$.

Note that the natural conformal frame

$$
\hat{E}_{A}= \begin{cases}\hat{E}_{a}^{+} & \text {for } A=a \\ \hat{E}_{\bar{a}}^{-} & \text {for } A=\bar{a}+d\end{cases}
$$

satisfies

$$
\left\langle\hat{E}_{A}, \hat{E}_{B}\right\rangle=\Phi^{2} \eta_{A B}, \quad \text { where } \quad \eta_{A B}=\left(\begin{array}{cc}
\eta_{a b} & 0 \\
0 & -\eta_{\bar{a} \bar{b}}
\end{array}\right),
$$

where the form of $\eta_{A B}$ differs from that used in (3.5). In this section, we will use this form of the metric $\eta_{A B}$ throughout. It is also important to note that we will adopt the convention that we will always raise and lower the $C_{+}$indices $a, b, c, \ldots$ with $\eta_{a b}$ and the $C_{-}$indices $\bar{a}, \bar{b}, \bar{c}, \ldots$ with $\eta_{\bar{a} \bar{b}}$, while we continue to raise and lower $2 d$ dimensional indices $A, B, C, \ldots$ with the $O(d, d)$ metric $\eta_{A B}$. Thus, for example we have

$$
\hat{E}^{A}=\left\{\begin{array}{ll}
\hat{E}^{+a} & \text { for } A=a \\
-\hat{E}^{-\bar{a}} & \text { for } A=\bar{a}+d
\end{array},\right.
$$

when we raise the $A$ index on the frame.

One can write a generic $O(p, q) \times O(q, p)$ structure explicitly as

$$
\begin{aligned}
& \hat{E}_{a}^{+}=\mathrm{e}^{-2 \phi} \sqrt{-g}\left(\hat{e}_{a}^{+}+e_{a}^{+}+i_{\hat{e}_{a}^{+}} B\right), \\
& \hat{E}_{\bar{a}}^{-}=\mathrm{e}^{-2 \phi} \sqrt{-g}\left(\hat{e}_{\bar{a}}^{-}-e_{\bar{a}}^{-}+i_{\hat{e}_{\bar{a}}^{-}} B\right),
\end{aligned}
$$

where the fixed conformal factor in (4.2) is given by

$$
\Phi=\mathrm{e}^{-2 \phi} \sqrt{-g}
$$

and where $\left\{\hat{e}_{a}^{+}\right\}$and $\left\{\hat{e}_{\bar{a}}^{-}\right\}$, and their duals $\left\{e^{+a}\right\}$ and $\left\{e^{-\bar{a}}\right\}$, are two independent orthonormal frames for the metric $g$, so that

$$
\begin{gathered}
g=\eta_{a b} e^{+a} \otimes e^{+b}=\eta_{\bar{a} \bar{b}} e^{-\bar{a}} \otimes e^{-\bar{b}}, \\
g\left(\hat{e}_{a}^{+}, \hat{e}_{b}^{+}\right)=\eta_{a b}, \quad g\left(\hat{e}_{\bar{a}}^{-}, \hat{e}_{\bar{b}}^{-}\right)=\eta_{\bar{a} \bar{b}} .
\end{gathered}
$$


By this explicit construction we see that there is no topological obstruction to the existence of $O(p, q) \times O(q, p)$ structures.

In addition to the $O(p, q) \times O(q, p)$ invariant density (4.7) one can also construct the invariant generalised metric $G[2]$. It has the form

$$
G=\Phi^{-2}\left(\eta^{a b} \hat{E}_{a}^{+} \otimes \hat{E}_{b}^{+}+\eta^{\bar{a} \bar{b}} \hat{E}_{\bar{a}}^{-} \otimes \hat{E}_{\bar{b}}^{-}\right)
$$

In the coordinate frame we have the familiar expression

$$
G_{M N}=\frac{1}{2}\left(\begin{array}{cc}
g-B g^{-1} B & -B g^{-1} \\
g^{-1} B & g^{-1}
\end{array}\right)_{M N} .
$$

By construction, the pair $(G, \Phi)$ parametrise the coset $\left(O(d, d) \times \mathbb{R}^{+}\right) / O(p, q) \times O(q, p)$ where $p+q=d$.

Finally the $O(p, q) \times O(q, p)$ structure provides two additional chirality operators $\Gamma^{ \pm}$on $\operatorname{Spin}(d, d) \times \mathbb{R}^{+}$spinors which one can define as $[42,47,50]$

$$
\Gamma^{(+)}=\frac{1}{d !} \epsilon^{a_{1} \ldots a_{d}} \Gamma_{a_{1}} \ldots \Gamma_{a_{d}}, \quad \quad \Gamma^{(-)}=\frac{1}{d !} \epsilon^{\bar{a}_{1} \ldots \bar{a}_{d}} \Gamma_{\bar{a}_{1}} \ldots \Gamma_{\bar{a}_{d}}
$$

Using that, in the split frame, the Clifford action takes the form

$$
\Gamma_{a} \cdot \Psi^{(B)}=i_{\hat{e}_{a}^{+}} \Psi^{(B)}+e_{a}^{+} \wedge \Psi^{(B)}, \quad \Gamma_{\bar{a}} \cdot \Psi^{(B)}=i_{\hat{e}_{a}^{-}} \Psi^{(B)}-e_{a}^{-} \wedge \Psi^{(B)},
$$

these can be evaluated on the weighted n-form components of $\Psi$ as

$$
\Gamma^{(+)} \Psi_{(n)}^{(B)}=(-)^{[n / 2]} * \Psi_{(n)}^{(B)}, \quad \quad \Gamma^{(-)} \Psi_{(n)}^{(B)}=(-)^{d}(-)^{[n+1 / 2]} * \Psi_{(n)}^{(B)},
$$

and thus we have a generalisation of the Hodge dual on $\operatorname{Spin}(d, d) \times \mathbb{R}^{+}$spinors.

Since $G^{T} \eta G=\eta$, the generalised metric $G^{A}{ }_{B}$ is an element of $O(d, d)$ and one can easily check that $G^{2}=1$. Connecting to the discussion of [50], for even dimensions $d$, one has $G \in S O(d, d)$ and $\Gamma^{(-)}$is an element of $\operatorname{Spin}(d, d)$ satisfying

$$
\Gamma^{(-)} \Gamma^{A} \Gamma^{(-)-1}=G_{B}^{A} \Gamma^{B},
$$

so that $\Gamma^{(-)}$is a preimage of $G$ in the double covering map $\operatorname{Spin}(d, d) \rightarrow S O(d, d)$. In odd dimensions $d, \Gamma^{(+)}$is an element of $\operatorname{Pin}(d, d)$ which maps to $G \in O(d, d)$ under the double cover $\operatorname{Pin}(d, d) \rightarrow O(d, d)$.

\subsection{Torsion-free, compatible connections}

A generalised connection $D$ is compatible with the $O(p, q) \times O(q, p)$ structure $P \subset \tilde{F}$ if

$$
D G=0, \quad D \Phi=0,
$$


or equivalently, if the derivative acts only in the $O(p, q) \times O(q, p)$ sub-bundle so that for $W \in \Gamma(\tilde{E})$ given by

$$
W=w_{+}^{a} \hat{E}_{a}^{+}+w_{-}^{\bar{a}} \hat{E}_{\bar{a}}^{-}
$$

we have

$$
D_{M} W^{A}= \begin{cases}\partial_{M} w_{+}^{a}+\Omega_{M}^{a} w^{b} w_{+}^{b} & \text { for } A=a \\ \partial_{M} w_{-}^{\bar{a}}+\Omega_{M}{ }^{\bar{a}} w_{-}^{\bar{b}} & \text { for } A=\bar{a}\end{cases}
$$

with

$$
\Omega_{M a b}=-\Omega_{M b a}, \quad \Omega_{M \bar{a} \bar{b}}=-\Omega_{M \bar{b} \bar{a}} .
$$

In this subsection we will show, in analogy to the construction of the Levi-Civita connection, that

Given an $O(p, q) \times O(q, p)$ structure $P \subset \tilde{F}$ there always exists a torsionfree, compatible generalised connection D. However, it is not unique.

We can construct a compatible connection as follows. Let $\nabla$ be the Levi-Civita connection for the metric $g$. In terms of the two orthonormal bases we get two gauge equivalent spin-connections, so that if $v=v^{a} \hat{e}_{a}^{+}=v^{\bar{a}} \hat{e}_{\bar{a}}^{-} \in \Gamma(T M)$ we have

$$
\nabla_{\mu} v^{\nu}=\left(\partial_{\mu} v^{a}+\omega_{\mu}^{+a} b v^{b}\right)\left(\hat{e}_{a}^{+}\right)^{\nu}=\left(\partial_{\mu} v^{\bar{a}}+\omega_{\mu}^{-\bar{a}} \bar{b} v^{\bar{b}}\right)\left(\hat{e}_{\bar{a}}^{-}\right)^{\nu}
$$

We can then define, as in (3.39)

$$
D_{M}^{\nabla} W^{a}=\left\{\begin{array}{ll}
\nabla_{\mu} w_{+}^{a} & \text { for } M=\mu \\
0 & \text { for } M=\mu+d
\end{array}, \quad D_{M}^{\nabla} W^{\bar{a}}= \begin{cases}\nabla_{\mu} w_{-}^{\bar{a}} & \text { for } M=\mu \\
0 & \text { for } M=\mu+d\end{cases}\right.
$$

Since $\omega_{\mu a b}^{+}=-\omega_{\mu b a}^{+}$and $\omega_{\mu \bar{a} \bar{b}}^{-}=-\omega_{\mu \bar{b} \bar{a}}^{-}$, by construction, this generalised connection is compatible with the $O(p, q) \times O(q, p)$ structure.

However $D^{\nabla}$ is not torsion-free. To see this we note that, comparing with (3.19), when we choose the two orthonormal frames to be aligned so $e_{a}^{+}=e_{a}^{-}=e_{a}$ we have

$$
W=w_{+}^{a} \hat{E}_{a}^{+}+w_{-}^{\bar{a}} \hat{E}_{\bar{a}}^{-}=\left(w_{+}^{a}+w_{-}^{a}\right) \hat{E}_{a}+\left(w_{+a}-w_{-a}\right) E^{a}
$$

and the two definitions of $D^{\nabla}$ in (3.39) and (4.20) agree. Hence from (3.50) we have the non-zero torsion components

$$
T_{1}=-4 H, \quad T_{2}=-4 \mathrm{~d} \phi .
$$

To construct a torsion-free compatible connection we simply modify $D^{\nabla}$. A generic generalised connection $D$ can be always be written as

$$
D_{M} W^{A}=D_{M}^{\nabla} W^{A}+\Sigma_{M}^{A}{ }_{B} W^{B} .
$$


If $D$ is compatible with the $O(p, q) \times O(q, p)$ structure then we have $\Sigma_{M}{ }^{a} \bar{b}=\Sigma_{M}{ }^{\bar{a}}{ }_{b}=0$ and

$$
\Sigma_{M a b}=-\Sigma_{M b a}, \quad \Sigma_{M \bar{a} \bar{b}}=-\Sigma_{M \bar{b} \bar{a}} .
$$

By definition, the generalised torsion components of $D$ are then given by

$$
\left(T_{1}\right)_{A B C}=-4 H_{A B C}-3 \Sigma_{[A B C]}, \quad\left(T_{2}\right)_{A}=-4 \mathrm{~d} \phi_{A}-\Sigma_{C}{ }_{A} .
$$

The components $H^{A B C}$ and $\mathrm{d} \phi^{A}$ are the components in frame indices of the corresponding forms under the embeddings $T^{*} M \hookrightarrow E$ and $\Lambda^{3} T^{*} M \hookrightarrow \Lambda^{3} E$. Given

$$
\mathrm{d} x^{\mu}=\frac{1}{2} \Phi^{-1}\left(\hat{e}_{a}^{+\mu} \hat{E}^{+a}-\hat{e}_{\bar{a}}^{-\mu} \hat{E}^{-\bar{a}}\right)
$$

we have, for instance,

$$
\mathrm{d} \phi=\frac{1}{2} \partial_{a} \phi\left(\Phi^{-1} \hat{E}^{+a}\right)-\frac{1}{2} \partial_{\bar{a}} \phi\left(\Phi^{-1} \hat{E}^{-\bar{a}}\right)
$$

where there is a similar decomposition of $H$ under

$$
\Lambda^{3} T^{*} M \hookrightarrow \Lambda^{3} E \simeq \Lambda^{3} C_{+} \oplus\left(\Lambda^{2} C_{+} \otimes C_{-}\right) \oplus\left(C_{+} \otimes \Lambda^{2} C_{-}\right) \oplus \Lambda^{3} C_{-}
$$

Note also that the middle index on $\Sigma_{[A B C]}$ in equation (4.25) has also been lowered with this $\eta_{A B}$ which introduces some signs. The result is that the components are

$$
\mathrm{d} \phi_{A}=\left\{\begin{array}{ll}
\frac{1}{2} \partial_{a} \phi & A=a \\
\frac{1}{2} \partial_{\bar{a}} \phi & A=\bar{a}+d
\end{array}, \quad H_{A B C}= \begin{cases}\frac{1}{8} H_{a b c} & (A, B, C)=(a, b, c) \\
\frac{1}{8} H_{a b \bar{c}} & (A, B, C)=(a, b, \bar{c}+d) \\
\frac{1}{8} H_{a \bar{b} \bar{c}} & (A, B, C)=(a, \bar{b}+d, \bar{c}+d) \\
\frac{1}{8} H_{\bar{a} \bar{b} \bar{c}} & (A, B, C)=(\bar{a}+d, \bar{b}+d, \bar{c}+d)\end{cases}\right.
$$

and that setting the torsion of $D$ to zero is equivalent to

$$
\begin{aligned}
& \Sigma_{[a b c]}=-\frac{1}{6} H_{a b c}, \quad \Sigma_{\bar{a} b c}=-\frac{1}{2} H_{\bar{a} b c}, \quad \Sigma_{a b}^{a}=-2 \partial_{b} \phi, \\
& \Sigma_{[\bar{a} \bar{b} \bar{c}]}=+\frac{1}{6} H_{\bar{a} \bar{b} \bar{c}}, \quad \Sigma_{a \bar{b} \bar{c}}=+\frac{1}{2} H_{a \bar{b} \bar{c}}, \quad \Sigma_{\bar{a}} \bar{a}_{\bar{b}}=-2 \partial_{\bar{b}} \phi .
\end{aligned}
$$

Thus we can always find a torsion-free compatible connection but clearly these conditions do not determine $D$ uniquely. Specifically, one finds

$$
\begin{aligned}
& D_{a} w_{+}^{b}=\nabla_{a} w_{+}^{b}-\frac{1}{6} H_{a}{ }^{b}{ }_{c} w_{+}^{c}-\frac{2}{9}\left(\delta_{a}{ }^{b} \partial_{c} \phi-\eta_{a c} \partial^{b} \phi\right) w_{+}^{c}+A_{a}^{+b}{ }_{c} w_{+}^{c}, \\
& D_{\bar{a}} w_{+}^{b}=\nabla_{\bar{a}} w_{+}^{b}-\frac{1}{2} H_{\bar{a}{ }^{b}{ }^{b} w_{+}^{c},} \\
& D_{a} w_{-}^{\bar{b}}=\nabla_{a} w_{-}^{\bar{b}}+\frac{1}{2} H_{a}^{\bar{b}} w_{-}^{\bar{c}}, \\
& D_{\bar{a}} w_{-}^{\bar{b}}=\nabla_{\bar{a}} w_{-}^{\bar{b}}+\frac{1}{6} H_{\bar{a} \bar{c}}^{\bar{b}} w_{-}^{\bar{c}}-\frac{2}{9}\left(\delta_{\bar{a}}{ }^{\bar{b}} \partial_{\bar{c}} \phi-\eta_{\bar{a} \bar{c}} \partial^{\bar{b}} \phi\right) w_{-}^{\bar{c}}+A_{\bar{a}}^{-\bar{b} \bar{c}} w_{-}^{\bar{c}},
\end{aligned}
$$


where the undetermined tensors $A^{ \pm}$satisfy

$$
\begin{aligned}
& A_{a b c}^{+}=-A_{a c b}^{+}, \quad A_{[a b c]}^{+}=0, \quad A_{a b}^{+a}=0, \\
& A_{\bar{a} \bar{b} \bar{c}}^{-}=-A_{\bar{a} \bar{c} \bar{b}}^{-}, \quad A_{[\bar{a} \bar{b} \bar{c}]}^{-}=0, \quad A_{\bar{a}}^{-\bar{a}_{\bar{b}}}=0,
\end{aligned}
$$

and hence do not contribute to the torsion.

\subsection{Unique operators and generalised $O(p, q) \times O(q, p)$ curvatures}

The fact that the $O(p, q) \times O(q, p)$ structure and torsion conditions are not sufficient to specify a unique generalised connection might raise ambiguities which could pose a problem for the applications to supergravity we are ultimately interested in. However, we will now show that it is still possible to find differential expressions that are independent of the chosen $D$, by forming $O(p, q) \times O(q, p)$ covariant operators which do not depend on the undetermined components $A^{ \pm}$. For example, by examining (4.31) we already see that

$$
\begin{aligned}
& D_{\bar{a}} w_{+}^{b}=\nabla_{\bar{a}} w_{+}^{b}-\frac{1}{2} H_{\bar{a}{ }^{b}{ }^{b} w_{+}^{c},}, \\
& D_{a} w_{-}^{\bar{b}}=\nabla_{a} w_{-}^{\bar{b}}+\frac{1}{2} H_{a}^{\bar{b}} w_{-}^{\bar{c}},
\end{aligned}
$$

have no dependence on $A^{ \pm}$and so are unique. We find that this is also true for

$$
\begin{aligned}
& D_{a} w_{+}^{a}=\nabla_{a} w_{+}^{a}-2\left(\partial_{a} \phi\right) w_{+}^{a}, \\
& D_{\bar{a}} w_{-}^{\bar{a}}=\nabla_{\bar{a}} w_{-}^{\bar{a}}-2\left(\partial_{\bar{a}} \phi\right) w_{-}^{\bar{a}} .
\end{aligned}
$$

Anticipating our application to supergravity, we will be especially interested in writing formulae for $\operatorname{Spin}(p, q)$ spinors, so let us now assume that we have a $\operatorname{Spin}(p, q) \times$ $\operatorname{Spin}(q, p)$ structure. If $S\left(C_{ \pm}\right)$are then the spinor bundles associated to the sub-bundles $C_{ \pm}, \gamma^{a}$ and $\gamma^{\bar{a}}$ the corresponding gamma matrices and $\epsilon^{ \pm} \in \Gamma\left(S\left(C_{ \pm}\right)\right)$, we have that by definition a generalised connection acts as

$$
\begin{aligned}
& D_{M} \epsilon^{+}=\partial_{M} \epsilon^{+}+\frac{1}{4} \Omega_{M}^{a b} \gamma_{a b} \epsilon^{+}, \\
& D_{M} \epsilon^{-}=\partial_{M} \epsilon^{-}+\frac{1}{4} \Omega_{M}{ }^{\bar{a} \bar{b}} \gamma_{\bar{a} \bar{b}} \epsilon^{-} .
\end{aligned}
$$

There are four operators which can be built out of these derivatives that are uniquely determined

$$
\begin{aligned}
D_{\bar{a}} \epsilon^{+} & =\left(\nabla_{\bar{a}}-\frac{1}{8} H_{\bar{a} b c} \gamma^{b c}\right) \epsilon^{+}, \\
D_{a} \epsilon^{-} & =\left(\nabla_{a}+\frac{1}{8} H_{a \bar{b} \bar{c}} \gamma^{\bar{b} \bar{c}}\right) \epsilon^{-}, \\
\gamma^{a} D_{a} \epsilon^{+} & =\left(\gamma^{a} \nabla_{a}-\frac{1}{24} H_{a b c} \gamma^{a b c}-\gamma^{a} \partial_{a} \phi\right) \epsilon^{+}, \\
\gamma^{\bar{a}} D_{\bar{a}} \epsilon^{-} & =\left(\gamma^{\bar{a}} \nabla_{\bar{a}}+\frac{1}{24} H_{\bar{a} \bar{b} \bar{c}} \gamma^{\bar{a} \bar{b} \bar{c}}-\gamma^{\bar{a}} \partial_{\bar{a}} \phi\right) \epsilon^{-} .
\end{aligned}
$$


The first two expressions follow directly from (4.33). In the final two expressions, there is an elegant cancellation from $\gamma^{a} \gamma^{b c}=\gamma^{a b c}+\eta^{a b} \gamma^{c}-\eta^{a c} \gamma^{b}$ which removes the terms involving $A^{ \pm}$.

The restriction that expressions involving generalised connections be determined unambiguously, irrespective of the particular $D$, now serves as a selection criteria for constructing new generalised objects. In particular, when defining a generalised notion of curvature, we find that even though we can actually build a tensorial $O(p, q) \times$ $O(q, p)$ generalised Riemann curvature - by following the example in section 3.4 .3 and taking $C_{1}=C_{ \pm}$and $C_{2}=C_{\mp}$ so that the index structure would be $\left(R_{a \bar{b} d}^{c}, R_{a \bar{b} \bar{d}}{ }_{\bar{c}}^{\bar{d}}\right)$ and $\left(R_{\bar{a} b d}^{c}, R_{\bar{a} b \bar{d}}^{\bar{c}}\right)$ - it would not result in a uniquely determined object. However, we can use combinations of (4.33) and (4.34) to define the corresponding generalised Ricci tensor as

$$
R_{a \bar{b}} w_{+}^{a}=\left[D_{a}, D_{\bar{b}}\right] w_{+}^{a}
$$

or $\mathrm{as}^{8}$

$$
R_{\bar{a} b} w_{-}^{\bar{a}}=\left[D_{\bar{a}}, D_{b}\right] w_{-}^{\bar{a}} .
$$

Note that the index contractions are precisely what is needed to guarantee uniqueness.

It is not possible to contract the remaining two indices in the generalised Ricci. Nonetheless, there does exist a notion of generalised scalar curvature, but to define it we need the help of spinors and the operators in (4.36). We can obtain the generalised Ricci again from either

$$
\begin{aligned}
& \frac{1}{2} R_{a \bar{b}} \gamma^{a} \epsilon^{+}=\left[\gamma^{a} D_{a}, D_{\bar{b}}\right] \epsilon^{+}, \\
& \frac{1}{2} R_{\bar{a} b} \gamma^{\bar{a}} \epsilon^{-}=\left[\gamma^{\bar{a}} D_{\bar{a}}, D_{b}\right] \epsilon^{-} .
\end{aligned}
$$

However, now we also find a generalised curvature scalar

$$
-\frac{1}{4} S \epsilon^{+}=\left(\gamma^{a} D_{a} \gamma^{b} D_{b}-D^{\bar{a}} D_{\bar{a}}\right) \epsilon^{+},
$$

or alternatively,

$$
-\frac{1}{4} S \epsilon^{-}=\left(\gamma^{\bar{a}} D_{\bar{a}} \gamma^{\bar{b}} D_{\bar{b}}-D^{a} D_{a}\right) \epsilon^{-} .
$$

Again, note the need to use the correct combinations of the operators in these definitions so that all the undetermined components drop out.

The fact that $S$ is indeed a scalar and not itself an operator might not be immediately apparent, so it is useful to work out the explicit form of these curvatures. This can be done by again choosing the two orthogonal frames to be aligned, $e_{a}^{+}=e_{a}^{-}$, to find

$$
R_{a b}=\mathcal{R}_{a b}-\frac{1}{4} H_{a c d} H_{b}^{c d}+2 \nabla_{a} \nabla_{b} \phi+\frac{1}{2} \mathrm{e}^{2 \phi} \nabla^{c}\left(\mathrm{e}^{-2 \phi} H_{c a b}\right)
$$

\footnotetext{
${ }^{8}$ Note that naively one might expect these definitions to give distinct tensors. However one can check that compatibility with the $O(p, q) \times O(q, p)$ structure means that the two agree.
} 
and for the scalar

$$
S=\mathcal{R}+4 \nabla^{2} \phi-4(\partial \phi)^{2}-\frac{1}{12} H^{2}
$$

From these expressions it is clear that we have obtained genuine tensors which are uniquely determined by the torsion conditions, as desired. Furthermore, comparing with $[14,15]$ we see that locally these are the same tensors that appear in Siegel's formulation. The expressions (4.42) and (4.43) also appear in the discussion of [23].

\section{$5 \quad$ Type II supergravity as $O(9,1) \times O(1,9)$ generalised gravity}

Having established the necessary elements of generalised geometry we need, let us now show how the dynamics and supersymmetry transformations of type II supergravity theories are encoded by an $O(9,1) \times O(1,9)$ structure with a compatible, torsion-free generalised connection. An outcome of this will be a formulation of type II supergravity with manifest local $O(9,1) \times O(1,9)$ symmetry.

In the following we will consider the full ten-dimensional supergraviy theory so that the relevant generalised structure is $O(10,10) \times \mathbb{R}^{+}$. However, one can equally well consider compactifications of theory of the form $\mathbb{R}^{9-d, 1} \times M$

$$
\mathrm{d} s_{10}^{2}=\mathrm{d} s^{2}\left(\mathbb{R}^{9-d, 1}\right)+\mathrm{d} s_{d}^{2},
$$

where $\mathrm{d} s^{2}\left(\mathbb{R}^{9-d, 1}\right)$ is the flat metric on $\mathbb{R}^{9-d, 1}$ and $\mathrm{d} s_{d}^{2}$ is a general metric on the $d$ dimensional manifold $M$. The relevant structure is then the $O(d) \times O(d) \subset O(d, d) \times \mathbb{R}^{+}$ generalised geometry on $M$. Below we will focus on the $O(10,10) \times \mathbb{R}^{+}$case. The compactification case follows essentially identically.

\subsection{NSNS and fermionic supergravity fields}

From the discussion of section 4.1 we see that an $O(9,1) \times O(1,9) \subset O(10,10) \times \mathbb{R}^{+}$ generalised structure is parametrised by a metric $g$ of signature $(9,1)$, a two-form $B$ patched as in (2.15) and a dilaton $\phi$, that is, at each point $x \in M$

$$
\{g, B, \phi\} \in \frac{O(10,10)}{O(9,1) \times O(1,9)} \times \mathbb{R}^{+} .
$$

Thus it precisely captures the NSNS bosonic fields of type II theories by packaging them into the generalised metric and conformal factor $(G, \Phi)$. As in [42], the infinitesimal bosonic symmetry transformation (2.20) is naturally encoded as the Dorfman derivative by $V=v+\lambda$

$$
\delta_{V} G=L_{V} G, \quad \delta_{V} \Phi=L_{V} \Phi
$$

and the algebra of these transformations is given by the Courant bracket. 
The type II fermionic degrees of freedom fall into spinor and vector-spinor representations of $\operatorname{Spin}(9,1) \times \operatorname{Spin}(1,9)^{9}$. Let $S\left(C_{+}\right)$and $S\left(C_{-}\right)$denote the $\operatorname{Spin}(9,1)$ spinor bundles associated to the sub-bundles $C_{ \pm}$write $\gamma^{a}$ and $\gamma^{\bar{a}}$ for the corresponding gamma matrices. Since we are in ten dimensions, we can further decompose into spinor bundles $S^{ \pm}\left(C_{+}\right)$and $S^{ \pm}\left(C_{-}\right)$of definite chirality under $\gamma^{(10)}$.

The gravitino degrees of freedom then correspond to

$$
\psi_{\bar{a}}^{+} \in \Gamma\left(C_{-} \otimes S^{\mp}\left(C_{+}\right)\right), \quad \psi_{a}^{-} \in \Gamma\left(C_{+} \otimes S^{+}\left(C_{-}\right)\right),
$$

where the upper sign on the chirality refers to type IIA and the lower to type IIB. Note that the vector and spinor parts of the gravitinos transform under different $\operatorname{Spin}(9,1)$ groups. For the dilatino degrees of freedom one has

$$
\rho^{+} \in \Gamma\left(S^{ \pm}\left(C_{+}\right)\right), \quad \rho^{-} \in \Gamma\left(S^{+}\left(C_{-}\right)\right),
$$

where again the upper and lower signs refer to IIA and IIB respectively. Similarly the supersymmetry parameters are sections

$$
\epsilon^{+} \in \Gamma\left(S^{\mp}\left(C_{+}\right)\right), \quad \epsilon^{-} \in \Gamma\left(S^{+}\left(C_{-}\right)\right) .
$$

In terms of the string spectrum these gravitino and dilatino representations just correspond to the explicit left- and right-moving fermionic states of the superstring and, in a supergravity context were discussed, for example, in [48].

\section{$5.2 \quad$ RR fields}

As is known from studying the action of T-duality, the RR field strengths transform as $\operatorname{Spin}(10,10)$ spinors $[24,25,48,49]$. Here, the patching $(2.17)$ of $A_{(i)}$ on $U_{i} \cap U_{j}$ implies that the polyform $F_{(i)}=\mathrm{d} A_{(i)}$ is patched as in (3.12), and hence, as generalised spinors,

$$
F \in \Gamma\left(S_{(1 / 2)}^{ \pm}\right),
$$

where the upper sign is for type IIA and the lower for type IIB. Furthermore, we see that the RR field strengths $F_{(n)}^{(B)}$ that appear in the supergravity (2.6) are simply $F$ expressed in a split frame as in (3.21)

$$
F^{(B)}=\mathrm{e}^{B_{(i)}} \wedge F_{(i)}=\mathrm{e}^{B_{(i)}} \wedge \sum_{n} \mathrm{~d} A_{(i)}^{(n-1)} .
$$

\footnotetext{
${ }^{9}$ Since the underlying manifold $M$ is assumed to possess a spin structure, we are free to promote $O(9,1) \times O(1,9)$ to $\operatorname{Spin}(9,1) \times \operatorname{Spin}(1,9)$. Here will ignore more complicated extended spin structures that can arise in generalised geometry as described in [9].
} 
Note that the additional gauge transformations $\mathrm{d} \hat{\Lambda}$ in (2.17) imply that $A_{(i)}$ does not globally define a section of $S_{(1 / 2)}^{ \pm}$. "Geometrising" this additional gauge symmetry is the subject of $E_{d(d)}$ generalised geometry [8]. Since $A_{(i)}$ is still locally a generalised spinor on the patch $U_{i}$ we can perform the same operations on it as we do on $F$ in the remainder of this subsection.

Given the generalised metric structure, we can also write $F$ in terms of $\operatorname{Spin}(9,1) \times$ $\operatorname{Spin}(1,9)$ representations. One has the decomposition $\operatorname{Cliff}(10,10 ; \mathbb{R}) \simeq \operatorname{Cliff}(9,1 ; \mathbb{R}) \otimes$ $\operatorname{Cliff}(1,9 ; \mathbb{R})$ with

$$
\Gamma^{A}= \begin{cases}\gamma^{a} \otimes 1 & \text { for } A=a \\ \gamma^{(10)} \otimes \gamma^{\bar{a}} \gamma^{(10)} & \text { for } A=\bar{a}+d\end{cases}
$$

and hence we can identify ${ }^{10}$

$$
S_{(1 / 2)} \simeq S\left(C_{+}\right) \otimes S\left(C_{-}\right) .
$$

Using the spinor norm on $S\left(C_{-}\right)$we can equally well view $F \in \Gamma\left(S_{(1 / 2)}\right)$ as a map from section of $S\left(C_{-}\right)$to sections of $S\left(C_{+}\right)$. We denote the image under this isomorphism as

$$
F_{\#}: S\left(C_{-}\right) \rightarrow S\left(C_{+}\right) .
$$

We have that $F \in \Gamma\left(S\left(C_{+}\right) \otimes S\left(C_{-}\right)\right)$naturally has spin indices $F^{\alpha \bar{\alpha}}$, while $F_{\#}$ naturally has indices $F^{\alpha}$. The isomorphism simply corresponds to lowering an index with the Cliff $(9,1 ; \mathbb{R})$ intertwiner $C_{\bar{\alpha} \bar{\beta}}$. The conjugate map, $F_{\#}^{T}: S\left(C_{+}\right) \rightarrow S\left(C_{-}\right)$, is given by

$$
F_{\#}^{T}=\left(C F_{\#} C^{-1}\right)^{T}
$$

which corresponds to lowering the other index on $F^{\alpha \bar{\alpha}}$ and taking the transpose.

We now give the relations between the components of the $\operatorname{Spin}(d, d) \times \mathbb{R}^{+}$spinor in all relevant frames. Note first that if the bases are aligned so that $e^{+}=e^{-}=e$ then the $\operatorname{Spin}(9,1) \times \operatorname{Spin}(1,9)$ basis $(4.6)$ is a split conformal basis and we have a $\operatorname{Spin}(9,1) \subset \operatorname{Spin}(9,1) \times \operatorname{Spin}(1,9)$ structure. We can then use the isomorphism $\operatorname{Cliff}(9,1 ; \mathbb{R}) \simeq \Lambda^{\bullet} T^{*} M$ to write $F^{(B, \phi)}$ as a spinor bilinear

$$
\not^{(B, \phi)}=\sum_{n} \frac{1}{n !} F_{a_{1} \ldots a_{n}}^{(B, \phi)} \gamma^{a_{1} \ldots a_{n}}
$$

More generally if the frames are related by Lorentz transformations $e_{a}^{ \pm}=\Lambda_{a}^{ \pm b} e_{a}$ and we write $\Lambda^{ \pm}$for the corresponding $\operatorname{Spin}(9,1)$ transformations then we can define $F_{\#}$ explicitly as

$$
F_{\#}=\Lambda^{+} \not^{(B, \phi)}\left(\Lambda^{-}\right)^{-1},
$$

\footnotetext{
${ }^{10}$ In fact $S_{(p)} \simeq S\left(C_{+}\right) \otimes S\left(C_{-}\right)$for any $p$, but here we focus on the case of interest $p=\frac{1}{2}$
} 
which concretely realises the isomorphism between $F^{(B, \phi)}$ and $F_{\#}$.

This map can easily be inverted and so we can write the components of $F \in$ $\Gamma\left(S_{(1 / 2)}\right)$ in the coordinate frame as

$$
\begin{aligned}
F_{(i)} & =\mathrm{e}^{-B_{(i)}} \wedge F^{(B)}=\mathrm{e}^{-\phi} \mathrm{e}^{-B_{(i)}} \wedge F^{(B, \phi)} \\
& =\mathrm{e}^{-\phi} \mathrm{e}^{-B_{(i)}} \wedge \sum_{n}\left[\frac{1}{32(n !)}(-)^{[n / 2]} \operatorname{tr}\left(\gamma_{(n)}\left(\Lambda^{+}\right)^{-1} F_{\#} \Lambda^{-}\right)\right] .
\end{aligned}
$$

This chain of equalities relates the components of $F$ in all the frames we have discussed.

Finally, we note that the self-duality conditions satisfied by the RR field strengths $F \in \Gamma\left(S_{(1 / 2)}^{ \pm}\right)$become a chirality condition under the operator $\Gamma^{(-)}$defined in (4.11)

$$
\Gamma^{(-)} F=-F,
$$

as discussed in $[30,50]$.

\subsection{Supersymmetry variations}

We now show that the supersymmetry variations can be written in a simple, locally $\operatorname{Spin}(9,1) \times \operatorname{Spin}(1,9)$ covariant form using the torsion-free compatible connection $D$.

We start with the fermionic variations (2.14). Looking at the expressions (4.36), we see that the uniquely determined spinor operators allow us to write the supersymmetry variations compactly as

$$
\begin{aligned}
\delta \psi_{\bar{a}}^{+} & =D_{\bar{a}} \epsilon^{+}+\frac{1}{16} F_{\#} \gamma_{\bar{a}} \epsilon^{-}, \\
\delta \psi_{a}^{-} & =D_{a} \epsilon^{-}+\frac{1}{16} F_{\#}^{T} \gamma_{a} \epsilon^{+}, \\
\delta \rho^{+} & =\gamma^{a} D_{a} \epsilon^{+}, \\
\delta \rho^{-} & =\gamma^{\bar{a}} D_{\bar{a}} \epsilon^{-},
\end{aligned}
$$

where we have also used the results from the previous section to add the RR field strengths to the gravitino variations.

For the bosonic fields, we need the variation of a generic $\operatorname{Spin}(9,1) \times \operatorname{Spin}(1,9)$ frame (4.6). Note that this means defining the variation of a pair of orthonormal bases $\left\{e^{+a}\right\}$ and $\left\{e^{-\bar{a}}\right\}$ whereas the conventional supersymmetry variations (2.13) are given in terms of a single basis $\left\{e^{a}\right\}$. The only possibility, compatible with the $\operatorname{Spin}(9,1) \times$ $\operatorname{Spin}(1,9)$ representations of the fermions, is to take

$$
\begin{aligned}
& \tilde{\delta} \hat{E}_{a}^{+}=(\delta \log \Phi) \hat{E}_{a}^{+}-\left(\delta \Lambda_{a \bar{b}}^{+}\right) \hat{E}^{-\bar{b}}, \\
& \tilde{\delta} \hat{E}_{\bar{a}}^{-}=(\delta \log \Phi) \hat{E}_{\bar{a}}^{-}-\left(\delta \Lambda_{\bar{a} b}^{-}\right) \hat{E}^{+b},
\end{aligned}
$$

where

$$
\begin{aligned}
& \delta \Lambda_{a \bar{a}}^{+}=\bar{\epsilon}^{+} \gamma_{a} \psi_{\bar{a}}^{+}+\bar{\epsilon}^{-} \gamma_{\bar{a}} \psi_{a}^{-}, \\
& \delta \Lambda_{a \bar{a}}^{-}=\bar{\epsilon}^{+} \gamma_{a} \psi_{\bar{a}}^{+}+\bar{\epsilon}^{-} \gamma_{\bar{a}} \psi_{a}^{-},
\end{aligned}
$$


and

$$
\delta \log \Phi=-2 \delta \phi+\frac{1}{2} \delta \log (-g)=\bar{\epsilon}^{+} \rho^{+}+\bar{\epsilon}^{-} \rho^{-} .
$$

Note that the variation of the basis (5.18) is by construction orthogonal to the $\operatorname{Spin}(9,1) \times$ $\operatorname{Spin}(1,9)$ action. This is because it is impossible to construct an $\operatorname{Spin}(9,1) \times \operatorname{Spin}(1,9)$ tensor linear in $\psi_{\bar{a}}^{+}$and $\psi_{a}^{-}$with two indices of the same type, that is $L_{a b}^{+}$or $L_{\bar{a} \bar{b}}^{-}$.

The corresponding variations of the frames $\hat{e}^{ \pm}$are

$$
\begin{aligned}
& \tilde{\delta} e_{\mu}^{+a}=\bar{\epsilon}^{+} \gamma_{\mu} \psi^{+a}+\bar{\epsilon}^{-} \gamma^{a} \psi_{\mu}^{-}, \\
& \tilde{\delta} e_{\mu}^{-\bar{a}}=\bar{\epsilon}^{+} \gamma^{\bar{a}} \psi_{\mu}^{+}+\bar{\epsilon}^{-} \gamma_{\mu} \psi^{-\bar{a}},
\end{aligned}
$$

which both give

$$
\tilde{\delta} g_{\mu \nu}=2 \bar{\epsilon}^{+} \gamma_{(\mu} \psi_{\nu)}^{+}+2 \bar{\epsilon}^{-} \gamma_{(\mu} \psi_{\nu)}^{-},
$$

as required, but, when setting the frames equal so $e^{+a}=e^{a}$ and $e^{-\bar{a}}=e^{\bar{a}}$, differ by Lorentz transformations from the standard form (2.13)

$$
\begin{aligned}
& \tilde{\delta} e_{\mu}^{+a}=\delta e_{\mu}^{+a}-\left(\bar{\epsilon}^{+} \gamma^{a} \psi^{+b}-\bar{\epsilon}^{+} \gamma^{b} \psi^{+a}\right) e_{\mu b}^{+}, \\
& \tilde{\delta} e_{\mu}^{-\bar{a}}=\delta e_{\mu}^{+\bar{a}}-\left(\bar{\epsilon}^{-} \gamma^{\bar{a}} \psi^{-\bar{b}}-\bar{\epsilon} \gamma^{-} \psi^{\bar{b}}\right) e_{\mu \bar{b}}^{-} .
\end{aligned}
$$

This can also be expressed in terms of the generalised metric $G_{A B}$ as

$$
\delta G_{a \bar{a}}=\delta G_{\bar{a} a}=2\left(\bar{\epsilon}^{+} \gamma_{a} \psi_{\bar{a}}^{+}+\bar{\epsilon}^{-} \gamma_{\bar{a}} \psi_{a}^{-}\right)
$$

The variation of the RR potential $A$ can be written as a bispinor

$$
\frac{1}{16}\left(\delta A_{\#}\right)=\left(\gamma^{a} \epsilon^{+} \bar{\psi}_{a}^{-}-\rho^{+} \bar{\epsilon}^{-}\right) \mp\left(\psi_{\bar{a}}^{+} \bar{\epsilon}^{-} \gamma^{\bar{a}}+\epsilon^{+} \bar{\rho}^{-}\right),
$$

where the upper sign is for type IIA and the lower for type IIB.

\subsection{Equations of motion}

Finally, we rewrite the supergravity equations of motion (2.9) and (2.10) with local $\operatorname{Spin}(9,1) \times \operatorname{Spin}(1,9)$ covariance, using the generalised notions of curvature obtained in section 4.3 .

From the generalised Ricci tensor (4.42), we find that the equations of motion for $g$ and $B$ can be written as

$$
R_{a \bar{b}}+\frac{1}{16} \Phi^{-1}\left\langle F, \Gamma_{a \bar{b}} F\right\rangle=0,
$$

where we have made use of the Mukai pairing defined in (3.14) ${ }^{11}$ to introduce the RR fields in a $\operatorname{Spin}(9,1) \times \operatorname{Spin}(1,9)$ covariant manner.

\footnotetext{
${ }^{11}$ Note that $\left\langle F, \Gamma_{a \bar{b}} F\right\rangle \in \Gamma\left(\left(\operatorname{det} T^{*} M\right) \otimes C_{+} \otimes C_{-}\right)$so $\Phi^{-1}\left\langle F, \Gamma_{a \bar{b}} F\right\rangle \in \Gamma\left(C_{+} \otimes C_{-}\right)$
} 
The equation of motion for $\phi$ does not involve the RR fields, so it is simply given by the generalised scalar curvature (4.43)

$$
S=0
$$

Using definition (3.32) and equation (3.46) we can write the equation of motion for the RR fields in the familiar form

$$
\frac{1}{2} \Gamma^{A} D_{A} F=\mathrm{d} F=0
$$

where the first equality serves as a reminder that this definition of the exterior derivative is fully covariant under $\operatorname{Spin}(d, d) \times \mathbb{R}^{+}$.

We also have the bosonic pseudo-action (2.5) which takes the simple form ${ }^{12}$

$$
S_{B}=\frac{1}{2 \kappa^{2}} \int\left(\Phi S+\frac{1}{4}\left\langle F, \Gamma^{(-)} F\right\rangle\right)
$$

using the density $\Phi$. Note that the Mukai pairing is a top-form which can be directly integrated.

The fermionic action (2.8) is given by

$$
\begin{aligned}
S_{F}=-\frac{1}{2 \kappa^{2}} \int 2 \Phi\left[\bar{\psi}^{+\bar{a}} \gamma^{b} D_{b} \psi_{\bar{a}}^{+}+\bar{\psi}^{-a} \gamma^{\bar{b}} D_{\bar{b}} \psi_{a}^{-}\right. \\
+2 \bar{\rho}^{+} D_{\bar{a}} \psi^{+\bar{a}}+2 \bar{\rho}^{-} D_{a} \psi^{-a} \\
-\bar{\rho}^{+} \gamma^{a} D_{a} \rho^{+}-\bar{\rho}^{-} \gamma^{\bar{a}} D_{\bar{a}} \rho^{-} \\
\left.-\frac{1}{8}\left(\bar{\rho}^{+} F_{\#} \rho^{-}+\bar{\psi}_{\bar{a}}^{+} \gamma^{a} F_{\#} \gamma^{\bar{a}} \psi_{a}^{-}\right)\right] .
\end{aligned}
$$

Varying this with respect to the fermionic fields leads to the generalised geometry version of (2.10)

$$
\begin{aligned}
\gamma^{b} D_{b} \psi_{\bar{a}}^{+}-D_{\bar{a}} \rho^{+} & =+\frac{1}{16} \gamma^{b} F_{\#} \gamma_{\bar{a}} \psi_{b}^{-}, \\
\gamma^{\bar{b}} D_{\bar{b}} \psi_{a}^{-}-D_{a} \rho^{-} & =+\frac{1}{16} \gamma^{\bar{b}} F_{\#}^{T} \gamma_{a} \psi_{\bar{b}}^{+}, \\
\gamma^{a} D_{a} \rho^{+}-D^{\bar{a}} \psi_{\bar{a}}^{+} & =-\frac{1}{16} F_{\#} \rho^{-}, \\
\gamma^{\bar{a}} D_{\bar{a}} \rho^{-}-D^{a} \psi_{a}^{-} & =-\frac{1}{16} F_{\#}^{T} \rho^{+},
\end{aligned}
$$

and it is straightforward to verify that by applying a supersymmetry variation (5.17) we recover the bosonic equations of motion (5.26)-(5.28).

We have thus rewritten all the supergravity equations from section 2.1 in terms of torsion free generalised connections and therefore as manifestly covariant under local $\operatorname{Spin}(9,1) \times \operatorname{Spin}(1,9)$ transformations.

\footnotetext{
${ }^{12} \mathrm{Up}$ to integration by parts of the $\nabla^{2} \phi$ term
} 


\section{Conclusions and discussion}

Starting with a generalised tangent space with a $O(10,10) \times \mathbb{R}^{+}$structure, we have shown that type II supergravity can be understood as a gravitational theory for a $\operatorname{Spin}(9,1) \times \operatorname{Spin}(1,9)$ substructure. It is defined using a torsion-free compatible connection $D$, in direct analogy to conventional gravity with a Levi-Civita connection. Our reformulation includes the leading fermionic equations of motion and action, and all the supersymmetry variations. The theory has a local $\operatorname{Spin}(9,1) \times \operatorname{Spin}(1,9)$ covariance together with an extension of the diffeomorphism group by the $B$-field gauge transformations.

As we mentioned in the introduction, both in Siegel's formulation $[14,15]$ and in double field theory [16], if one requires the action to be gauge invariant, one imposes the condition that on each coordinate patch the fields are independent of half the doubled coordinates. Thus locally, the generalised geometry and double field theory descriptions are completely equivalent, and our reformulation also gives the fermionic equations of motion, action and the supersymmetry variations in double field theory.

The relation of our formalism to Siegel's is interesting since naively he uses a $G L(d, \mathbb{R}) \times G L(d, \mathbb{R})$ structure rather than a $O(p, q) \times O(q, p)$ structure. However, his $G L(d, \mathbb{R}) \times G L(d, \mathbb{R})$ connection is also required preserve the $O(d, d)$ metric and the volume measure ( $\Phi$ in our notation). Thus in fact, the connection is compatible with the common subgroup of $O(d, d) \times \mathbb{R}^{+}$and the appropriate embedding of $G L(d, \mathbb{R}) \times G L(d, \mathbb{R})$ in $G L(2 d, \mathbb{R})$, namely $O(p, q) \times O(q, p)$. This explains the agreement of our curvature tensors. Similarly the lack of covariance of Siegel's (modified) putative Riemann tensor is a reflection of the non-tensorial nature described in (3.52).

One of the most remarkable properties of the reformulation is that supersymmetry was not used in the construction of the connection $D$ and yet it has precisely the properties necessary for the supersymmetry algebra to close. For instance, from (5.24) and (5.17) we see that the double variation of generalised metric is just a Dorfman derivative $L_{V} G$, that is simply a diffeomorphism plus gauge transformation, precisely because $D$ is torsion-free. This is strongly suggestive of the fact that the construction has a natural supersymmetric extension.

As will be reported in [8], similar generalised geometrical constructions, using

$E_{d(d)} \times \mathbb{R}^{+}$structures, describe eleven-dimensional supergravity restricted to $d$-dimensional spacetimes. In fact, there is evidence that several pure supergravity theories, in varying dimensions, can be formulated this way. This leads to the question of why there is such a general relationship between supergravity and versions of generalised geometry and which types of structure groups can appear.

There are a number of other directions for which this formulation may prove useful. 
One is the description of higher-derivative correction terms to the theory, assuming the generalised structure is not broken. Another is the explicit construction of supergravity backgrounds, for instance as spaces with particular special structures on the generalised tangent space. One can also connect this work to that on non-geometrical backgrounds. For example, in [34] the NSNS action is rewritten in terms of a "non-geometrical" flux $Q$. In the formalism of this paper, this amounts to evaluating $S$ in a different frame from the standard split frame used in (4.43). One takes instead $\hat{E}_{a}=\mathrm{e}^{-2 \phi}(\operatorname{det} e) \hat{e}_{a}$ and $E^{a}=$ $\mathrm{e}^{-2 \phi}(\operatorname{det} e)\left(e^{a}+\beta \hat{e}^{a}\right)$ where $\beta$ is a bivector $\beta \in \Gamma\left(\Lambda^{2} T M\right)$. Locally, from a generalised geometrical perspective, these are equivalent. However, given the patching (3.2), the new frame is not, generically, globally defined in a conventional generalised geometry. The suggestion though is that on a non-geometrical background (patched for instance by a T-duality) it may be possible to make some global notion of such a frame.

Perhaps, indeed, the most interesting question is whether there can be any extension of the generalised geometrical picture described here relevant to such exotic string backgrounds. One that moves away from a conventional ten-dimensional manifold, while still retaining "geometrical" notions of, for example, connections and curvatures, as some sensible limit of the full string theory.

\section{Acknowledgments}

We would like to thank Mariana Graña and Chris Hull for helpful discussions. C. SC. is supported by an STFC PhD studentship. A. C. is supported by the Portuguese Fundação para a Ciência e a Tecnologia under grant SFRH/BD/43249/2008. D. W. also thanks CEA/Saclay and the Mitchell Institute for Fundamental Physics and Astronomy at Texas A\&M for hospitality during the completion of this work.

\section{A Supergravity Conventions}

Our conventions largely follow [40] but we include a list for completeness. The only difference which is not purely notational is that we take the opposite sign for the Riemann tensor, as discussed in appendix B. The metric has the mostly plus signature $(-++\cdots+)$. We use the indices $\mu, \nu, \lambda \ldots$ as the spacetime coordinate indices and $a, b, c \ldots$ for the tangent space indices. We take symmetrisation of indices with weight 
one. Our conventions for forms are

$$
\begin{aligned}
\omega_{(k)} & =\frac{1}{k !} \omega_{\mu_{1} \ldots \mu_{k}} \mathrm{~d} x^{\mu_{1}} \wedge \cdots \wedge \mathrm{d} x^{\mu_{k}} \\
\omega_{(k)} \wedge \eta_{(l)} & =\frac{1}{(k+l) !}\left(\frac{(k+l) !}{k ! ! !} \omega_{\left[\mu_{1} \ldots \mu_{k}\right.} \eta_{\left.\mu_{k+1} \ldots \mu_{k+l}\right]}\right) \mathrm{d} x^{\mu_{1}} \wedge \cdots \wedge \mathrm{d} x^{\mu_{k+l}} \\
* \omega_{(k)} & =\frac{1}{(10-k) !}\left(\frac{1}{k !} \sqrt{-g} \epsilon_{\mu_{1} \ldots \mu_{10-k} \nu_{1} \ldots \nu_{k}} \omega^{\nu_{1} \ldots \nu_{k}}\right) \mathrm{d} x^{\mu_{1}} \wedge \cdots \wedge \mathrm{d} x^{\mu_{10-k}}
\end{aligned}
$$

where $\epsilon_{01 \ldots 9}=-\epsilon^{01 \ldots 9}=+1$. The gamma matrices have

$$
\left\{\gamma^{\mu}, \gamma^{\nu}\right\}=2 g^{\mu \nu}, \quad \gamma^{\mu_{1} \ldots \mu_{k}}=\gamma^{\left[\mu_{1}\right.} \ldots \gamma^{\left.\mu_{k}\right]}
$$

and we use the anti-symmetric transpose intertwiner

$$
C \gamma^{\mu} C^{-1}=-\left(\gamma^{\mu}\right)^{T}, \quad C^{T}=-C
$$

to define the Majorana conjugate as $\bar{\epsilon}=\epsilon^{T} C$. This leads to the formulae

$$
\begin{aligned}
C \gamma^{\mu_{1} \ldots \mu_{k}} C^{-1} & =(-)^{[(k+1) / 2]}\left(\gamma^{\mu_{1} \ldots \mu_{k}}\right)^{T}, \\
\bar{\epsilon} \gamma^{\mu_{1} \ldots \mu_{k}} \chi & =(-)^{[(k+1) / 2]} \bar{\chi} \gamma^{\mu_{1} \ldots \mu_{k}} \epsilon,
\end{aligned}
$$

where in the second equation the spinors $\epsilon$ and $\chi$ are anti-commuting. The top gamma is defined as

$$
\gamma^{(10)}=\gamma^{0} \gamma^{1} \ldots \gamma^{9}=\frac{1}{10 !} \epsilon_{\mu_{1} \ldots \mu_{10}} \gamma^{\mu_{1} \ldots \mu_{10}}
$$

and this gives rise to the equation

$$
\gamma_{\mu_{1} \ldots \mu_{k}} \gamma^{(10)}=(-)^{[k / 2]} \frac{1}{(10-k) !} \sqrt{-g} \epsilon_{\mu_{1} \ldots \mu_{k} \nu_{1} \ldots \nu_{10-k}} \gamma^{\nu_{1} \ldots \nu_{10-k}}
$$

which is also commonly written as

$$
\gamma^{(k)} \gamma^{(10)}=(-)^{[k / 2]} * \gamma^{(10-k)}
$$

We use Dirac slash notation with weight one so that for $\Psi \in \Gamma\left(\Lambda^{\bullet} T^{*} M\right)$

$$
\Psi=\sum_{k} \frac{1}{k !} \Psi_{\mu_{1} \ldots \mu_{k}} \gamma^{\mu_{1} \ldots \mu_{k}}
$$

\section{B Metric structures, torsion and the Levi-Civita connection}

In this appendix we briefly review the basic geometry that goes into the construction of the Levi-Civita connection, as context for the corresponding generalised geometrical analogues. 
Let $M$ be a $d$-dimensional manifold. We write $\left\{\hat{e}_{a}\right\}$ for a basis of the tangent space $T_{x} M$ at $x \in M$ and $\left\{e^{a}\right\}$ be the dual basis of $T_{x}^{*} M$ satisfying $i_{\hat{e}_{a}} e^{b}=\delta_{a}{ }^{b}$. Recall that the frame bundle $F$ is the bundle of all bases $\left\{\hat{e}^{a}\right\}$ over $M$,

$$
F=\left\{\left(x,\left\{\hat{e}_{a}\right\}\right): x \in M \text { and }\left\{\hat{e}_{a}\right\} \text { is a basis for } T_{x} M\right\} .
$$

On each fibre of $F$ there is an action of $A^{a}{ }_{b} \in G L(d, \mathbb{R})$, given $v=v^{a} \hat{e}_{a} \in \Gamma\left(T_{x} M\right)$,

$$
v^{a} \mapsto v^{\prime a}=A^{a}{ }_{b} v^{b}, \quad \hat{e}_{a} \mapsto \hat{e}_{a}^{\prime}=\hat{e}_{b}\left(A^{-1}\right)^{b}{ }_{a} .
$$

giving $F$ the structure of a $G L(d, \mathbb{R})$ principal bundle.

The Lie derivative $\mathcal{L}_{v}$ encodes the effect of an infinitesimal diffeomorphism. On a vector field $w$ it is equal to the Lie bracket

$$
\mathcal{L}_{v} w=-\mathcal{L}_{w} v=[v, w],
$$

while on a general tensor field $\alpha$ one has, in coordinate indices,

$$
\begin{aligned}
\mathcal{L}_{v} \alpha_{\nu_{1} \ldots \nu_{q}}^{\mu_{1} \ldots \mu_{p}}=v^{\mu} & \partial_{\mu} \alpha_{\nu_{1} \ldots \nu_{q}}^{\mu_{1} \ldots \mu_{p}} \\
& +\left(\partial_{\mu} v^{\mu_{1}}\right) \alpha_{\nu_{1} \ldots \nu_{q}}^{\mu \mu_{2} \ldots \mu_{p}}+\cdots+\left(\partial_{\mu} v^{\mu_{p}}\right) \alpha_{\nu_{1} \ldots \nu_{q}}^{\mu_{1} \ldots \mu_{p-1} \mu} \\
& -\left(\partial_{\nu_{1}} v^{\mu}\right) \alpha_{\mu \nu_{2} \ldots \nu_{q}}^{\mu_{1} \ldots \mu_{p}}-\cdots-\left(\partial_{\nu_{q}} v^{\mu}\right) \alpha_{\nu_{1} \ldots \nu_{q-1} \mu}^{\mu_{1} \ldots \mu_{p}}
\end{aligned}
$$

Note that the terms on the second and third lines can be viewed as the adjoint action of the $\mathfrak{g l}(d, \mathbb{R})$ matrix $a^{\mu}{ }_{\nu}=\partial_{\nu} v^{\mu}$ on the particular tensor field $\alpha$. This form will have an analogous expression when we come to generalised geometry.

Let $\nabla_{\mu} v^{\nu}=\partial_{\mu} v^{\nu}+\omega_{\mu}{ }_{\lambda}{ } v^{\lambda}$ be a general connection on $T M$. The torsion $T \in$ $\Gamma\left(T M \otimes \Lambda^{2} T^{*} M\right)$ of $\nabla$ is defined by

$$
T(v, w)=\nabla_{v} w-\nabla_{w} v-[v, w] .
$$

or concretely, in coordinate indices,

$$
T^{\mu}{ }_{\nu \lambda}=\omega_{\nu}{ }^{\mu}{ }_{\lambda}-\omega_{\lambda}{ }^{\mu}{ }_{\nu}
$$

while, in a general basis where $\nabla_{\mu} v^{a}=\partial_{\mu} v^{a}+\omega_{\mu}^{a} v^{b}$, one has

$$
T_{b c}^{a}=\omega_{b c}^{a}-\omega_{c}^{a} b+\left[\hat{e}_{b}, \hat{e}_{c}\right]^{a} .
$$

Since again it has a natural generalised geometric analogue, it is useful to equivalently define the torsion in terms of the Lie derivative. If $\mathcal{L}_{v}^{\nabla} \alpha$ is the analogue of the Lie derivative (B.4) but with $\partial$ replaced by $\nabla$, and $\left(i_{v} T\right)^{\mu}{ }_{\nu}=v^{\lambda} T_{\lambda \nu}^{\mu}$ then

$$
\left(i_{v} T\right) \alpha=\mathcal{L}_{v}^{\nabla} \alpha-\mathcal{L}_{v} \alpha,
$$


where we view $i_{v} T$ as a section of the $\mathfrak{g l}(d, \mathbb{R})$ adjoint bundle acting on the given tensor field $\alpha$.

The curvature of a connection $\nabla$ is given by the Riemann tensor $\mathcal{R} \in \Gamma\left(\Lambda^{2} T^{*} M \otimes\right.$ $\left.T M \otimes T^{*} M\right)$, defined by

$$
\begin{aligned}
\mathcal{R}(u, v) w & =\left[\nabla_{u}, \nabla_{v}\right] w-\nabla_{[u, v]} w \\
\mathcal{R}_{\mu \nu}{ }^{\lambda}{ }_{\rho} v^{\rho} & =\left[\nabla_{\mu}, \nabla_{\nu}\right] v^{\lambda}-T_{\mu \nu}^{\rho} \nabla_{\rho} v^{\lambda} .
\end{aligned}
$$

The Ricci tensor is the trace of the Riemann curvature

$$
\mathcal{R}_{\mu \nu}=\mathcal{R}_{\lambda \mu \nu}{ }^{\lambda}
$$

If the manifold admits a metric $g$ then the Ricci scalar is defined by

$$
\mathcal{R}=g^{\mu \nu} \mathcal{R}_{\mu \nu}
$$

A $G$-structure is a principal sub-bundle $P \subset F$ with fibre $G$. In the case of the metric $g$, the $G=O(d)$ sub-bundle is formed by the set of orthonormal bases

$$
P=\left\{\left(x,\left\{\hat{e}_{a}\right\}\right) \in F: g\left(\hat{e}_{a}, \hat{e}_{b}\right)=\delta_{a b}\right\},
$$

related by an $O(d) \subset G L(d, \mathbb{R})$ action. (A Lorentzian defines a $O(d-1,1)$-structure and $\delta_{a b}$ is replaced by $\eta_{a b}$.) At each point $x \in M$, the metric defines a point in the coset space

$$
\left.g\right|_{x} \in G L(d, \mathbb{R}) / O(d) .
$$

In general the existence of a $G$-structure can impose topological conditions on the manifold, since it implies that the tangent space can be patched using only $G \subset G L(d, \mathbb{R})$ transition functions. (For example, for even $d$, if $G=G L(d / 2, \mathbb{C})$, the manifold must admit an almost complex structure, while for $G=S L(d, \mathbb{R})$ it must be orientable.) However, for $O(d)$ there is no such restriction.

A connection $\nabla$ is compatible with a $G$-structure $P \subset E$ if the corresponding connection of the principal bundle $E$ reduces to a connection on $P$. This means that, given a basis $\left\{\hat{e}_{a}\right\}$, one has a set of connection one-forms $\omega^{a}{ }_{b}$ taking values in the adjoint representation of $G$ given by

$$
\nabla_{\partial / \partial x^{\mu}} \hat{e}_{a}=\omega_{\mu}^{b}{ }^{b} \hat{e}_{b} .
$$

For a metric structure this is equivalent to the condition $\nabla g=0$. If there exists a torsion-free compatible connection, the $G$-structure is said to be torsion-free or equivalently integrable (to first order). In general this can further restrict the structure, for instance in the case of $G L(d / 2, \mathbb{C})$ it is equivalent to the existence of a complex structure (satisfying the Nijenhuis condition). However, for a metric structure no further conditions are implied, and furthermore the torsion-free, compatible connection, namely the Levi-Civita connection, is unique. 


\section{References}

[1] N. Hitchin, "Generalized Calabi-Yau manifolds," Quart. J. Math. Oxford Ser. 54, 281 (2003) [arXiv:math.dg/0209099].

[2] M. Gualtieri, "Generalized Complex Geometry," Oxford University DPhil thesis (2004) [arXiv:math.DG/0401221] and [arXiv:math.DG/0703298].

[3] D. Roytenberg, "Courant algebroids, derived brackets and even symplectic supermanifolds", Ph.D. Thesis, U.C. Berkeley, arXiv:math/9910078 .

[4] M. Grana, R. Minasian, M. Petrini and A. Tomasiello, "Supersymmetric backgrounds from generalized Calabi-Yau manifolds," JHEP 0408 (2004) 046 [arXiv:hep-th/0406137].

M. Grana, R. Minasian, M. Petrini and A. Tomasiello, "Generalized structures of $N=1$ vacua," JHEP 0511 (2005) 020 [arXiv:hep-th/0505212].

[5] C. Jeschek and F. Witt, "Generalised $G_{2}$-structures and type IIB superstrings," JHEP 0503 (2005) 053 [arXiv:hep-th/0412280].

[6] P. Berglund and P. Mayr, "Non-perturbative superpotentials in F-theory and string duality," arXiv:hep-th/0504058.

[7] U. Lindstrom, "Generalized $\mathrm{N}=(2,2)$ supersymmetric nonlinear sigma models," Phys. Lett. B 587, 216 (2004) [arXiv:hep-th/0401100], U. Lindstrom, R. Minasian, A. Tomasiello and M. Zabzine, "Generalized complex manifolds and supersymmetry," Commun. Math. Phys. 257, 235 (2005) [arXiv:hep-th/0405085],

A. Kapustin, "Topological strings on noncommutative manifolds," Int. J. Geom. Meth. Mod. Phys. 1, 49 (2004) [arXiv:hep-th/0310057],

A. Kapustin and Y. Li, "Topological sigma-models with $H$-flux and twisted generalized complex manifolds," arXiv:hep-th/0407249.

[8] A. Coimbra, C. Strickland-Constable, D. Waldram, to appear.

[9] C. M. Hull, "Generalised Geometry for M-Theory," JHEP 0707, 079 (2007) [arXiv:hep-th/0701203].

[10] P. P. Pacheco, D. Waldram, "M-theory, exceptional generalised geometry and superpotentials," JHEP 0809, 123 (2008). [arXiv:0804.1362 [hep-th]].

[11] B. de Wit and H. Nicolai, " $D=11$ Supergravity With Local $S U(8)$ Invariance," Nucl. Phys. B 274, 363 (1986).

[12] H. Nicolai, " $D=11$ Supergravity with Local $S O(16)$ Invariance," Phys. Lett. B 187, 316 (1987). 
[13] K. Koepsell, H. Nicolai and H. Samtleben, "An exceptional geometry for $d=11$ supergravity?," Class. Quant. Grav. 17, 3689 (2000) [arXiv:hep-th/0006034].

[14] W. Siegel, "Two vierbein formalism for string inspired axionic gravity," Phys. Rev. D47 (1993) 5453-5459. [hep-th/9302036].

[15] W. Siegel, "Superspace duality in low-energy superstrings," Phys. Rev. D48 (1993) 2826-2837. [hep-th/9305073].

[16] C. Hull, B. Zwiebach, "Double Field Theory," JHEP 0909 (2009) 099. [arXiv:0904.4664 [hep-th]].

[17] C. M. Hull, "A geometry for non-geometric string backgrounds," JHEP 0510, 065 (2005) [arXiv:hep-th/0406102].

[18] C. Hull, B. Zwiebach, "The Gauge algebra of double field theory and Courant brackets," JHEP 0909 (2009) 090. [arXiv:0908.1792 [hep-th]].

[19] O. Hohm, C. Hull, B. Zwiebach, "Background independent action for double field theory," JHEP 1007 (2010) 016. [arXiv:1003.5027 [hep-th]].

[20] O. Hohm, C. Hull, B. Zwiebach, "Generalized metric formulation of double field theory," JHEP 1008 (2010) 008. [arXiv:1006.4823 [hep-th]].

[21] S. K. Kwak, "Invariances and Equations of Motion in Double Field Theory," JHEP 1010 (2010) 047. [arXiv:1008.2746 [hep-th]].

[22] O. Hohm, S. K. Kwak, "Frame-like Geometry of Double Field Theory," J. Phys. A A44 (2011) 085404. [arXiv:1011.4101 [hep-th]].

[23] I. Jeon, K. Lee, J. -H. Park, "Differential geometry with a projection: Application to double field theory," JHEP 1104 (2011) 014. [arXiv:1011.1324 [hep-th]],

I. Jeon, K. Lee, J. -H. Park, "Stringy differential geometry, beyond Riemann," Phys. Rev. D84, 044022 (2011). [arXiv:1105.6294 [hep-th]],

[24] D. Brace, B. Morariu, B. Zumino, "T duality and Ramond-Ramond backgrounds in the matrix model," Nucl. Phys. B549, 181-193 (1999). [hep-th/9811213].

[25] M. Fukuma, T. Oota, H. Tanaka, "Comments on T dualities of Ramond-Ramond potentials on tori," Prog. Theor. Phys. 103 (2000) 425-446. [hep-th/9907132].

[26] C. Hull, private communication.

[27] P. C. West, "E $E_{11}$ and M theory," Class. Quant. Grav. 18, 4443-4460 (2001). [hep-th/0104081], P. C. West, " $E_{11}, S L(32)$ and central charges," Phys. Lett. B575, 333-342 (2003). [hep-th/0307098].

[28] P. C. West, "The IIA, IIB and eleven-dimensional theories and their common $E_{11}$ origin," Nucl. Phys. B693 (2004) 76-102. [hep-th/0402140]. 
[29] P. West, " $E_{11}$, generalised space-time and IIA string theory," Phys. Lett. B696 (2011) 403-409. [arXiv:1009.2624 [hep-th]].

[30] A. Rocen, P. West, " $E_{11}$, generalised space-time and IIA string theory: the R-R sector," [arXiv:1012.2744 [hep-th]].

[31] D. S. Berman, M. J. Perry, "Generalized Geometry and M theory," JHEP 1106, 074 (2011). [arXiv:1008.1763 [hep-th]],

D. S. Berman, H. Godazgar, M. J. Perry, " $S O(5,5)$ duality in M-theory and generalized geometry," Phys. Lett. B700 (2011) 65-67. [arXiv:1103.5733 [hep-th]].

[32] O. Hohm, "On factorizations in perturbative quantum gravity," JHEP 1104 (2011) 103. [arXiv:1103.0032 [hep-th]].

[33] N. B. Copland, "Connecting T-duality invariant theories," [arXiv:1106.1888 [hep-th]].

[34] D. Andriot, M. Larfors, D. Lust, P. Patalong, "A ten-dimensional action for non-geometric fluxes," [arXiv:1106.4015 [hep-th]].

[35] D. C. Thompson, "Duality Invariance: From M-theory to Double Field Theory," JHEP 1108, 125 (2011) [arXiv:1106.4036 [hep-th]].

[36] Z. Chen, M. Stienon and P. Xu, "On Regular Courant Algebroids", arXiv:0909.0319.

[37] A. Alekseev and P. Xu, "Derived brackets and Courant algebroids", (2001). unpublished manuscript.

[38] I. T. Ellwood, "NS-NS fluxes in Hitchin's generalized geometry," JHEP 0712, 084 (2007) [arXiv:hep-th/0612100].

[39] M. Gualtieri, "Branes on Poisson varieties", in The Many Facets of Geometry, A Tribute to Nigel Hitchin, edited by Oscar Garcia-Prada, Jean Pierre Bourguignon and Simon Salamon, OUP, 2010, arXiv:0710.2719 [math.DG].

[40] E. Bergshoeff, R. Kallosh, T. Ortin, D. Roest, A. Van Proeyen, "New formulations of D = 10 supersymmetry and D8 - O8 domain walls," Class. Quant. Grav. 18, 3359-3382 (2001) [hep-th/0103233].

[41] N. J. Hitchin, "Lectures on special Lagrangian submanifolds," arXiv:math/9907034.

[42] M. Grana, R. Minasian, M. Petrini and D. Waldram, "T-duality, Generalized Geometry and Non-Geometric Backgrounds," JHEP 0904, 075 (2009) [arXiv:0807.4527 [hep-th]].

[43] C. Jeschek, F. Witt, "Generalised geometries, constrained critical points and Ramond-Ramond fields," [math/0510131 [math.DG]].

[44] I. Ya. Dorfman, "Dirac structures of integrable evolution equations" Physics Lett. A 125, 240 (1987).

[45] T. J. Courant, "Dirac manifolds", Trans. Amer. Math. Soc. 319, 631 (1990). 
[46] Y. Kosmann-Schwarzbach, "Derived brackets," Lett. Math. Phys. 69, 61 (2004) [arXiv:math/0312524].

[47] M. Gualtieri, "Generalized geometry and the Hodge decomposition", Lecture at the String Theory and Geometry workshop, August 2004, Oberwolfach, arXiv:math.dg/0409093.

[48] S. F. Hassan, "SO (d,d) transformations of Ramond-Ramond fields and space-time spinors," Nucl. Phys. B583 (2000) 431-453. [hep-th/9912236],

S. F. Hassan, "T duality, space-time spinors and RR fields in curved backgrounds," Nucl. Phys. B568 (2000) 145-161. [hep-th/9907152].

[49] C. M. Hull and P. K. Townsend, "Unity of superstring dualities," Nucl. Phys. B 438 (1995) 109 [arXiv:hep-th/9410167].

[50] O. Hohm, S. K. Kwak, B. Zwiebach, "Unification of Type II Strings and T-duality," [arXiv:1106.5452 [hep-th]].

O. Hohm, S. K. Kwak, B. Zwiebach, "Double Field Theory of Type II Strings," JHEP 1109, 013 (2011). [arXiv:1107.0008 [hep-th]]. 